\title{
HIERARCHICAL STRUCTURE FORMATION AND CHEMICAL EVOLUTION OF GALAXIES
}

\author{
Y.-Z. QIAN ${ }^{1}$ AND G. J. WasserburG ${ }^{2}$ \\ Received 2004 February 24; accepted 2004 May 19
}

\begin{abstract}
We present an analytical and phenomenological model for metal enrichment in halos based on hierarchical structure formation. This model assumes that astration of normal stellar populations along with Type II supernovae (SNe II) already occurs at very high redshift. It focuses on the regime of $[\mathrm{Fe} / \mathrm{H}]<-1$, in which SNe Ia are not major contributors. The regime of $[\mathrm{Fe} / \mathrm{H}]>-1$, in which SNe Ia are major contributors, is also discussed in general. For halos that are not disrupted by SN II explosions, the chemical evolution of the gas and stars is explicitly determined by the rate of gas infall as compared with the astration rate and the corresponding rate of metal production by SNe II per $\mathrm{H}$ atom in the gas. This model provides a good description of the data on $[\mathrm{Fe} / \mathrm{H}]$ for damped Ly $\alpha$ systems over a wide range of redshift, $0.5<z<5$. For all halos not disrupted by SN II explosions, if there is a cessation of gas infall, the metallicities of the stars follow a bimodal distribution. This distribution is characterized by a sharp peak at the value of $[\mathrm{Fe} / \mathrm{H}]$ corresponding to the time of infall cessation and by a broad peak at a higher value of $[\mathrm{Fe} / \mathrm{H}]$ corresponding to the subsequent period of astration during which the bulk of the remaining gas forms stars. Such a distribution can be compared with that observed for the Galactic halo stars. If the gas in a halo is rapidly lost on cessation of infall, then an assemblage of stars with a very sharply defined $[\mathrm{Fe} / \mathrm{H}]$ value will be left behind. This assemblage of stars may be accreted by a larger system and become a globular cluster of the larger system. We discuss the masses and metallicities of the globular clusters in this model considering the criterion for the onset of astration and possible disruption of low-mass halos by SN II explosions. The implications of possible globular clusters with very low metallicities of $[\mathrm{Fe} / \mathrm{H}] \lesssim-3$ are also discussed.
\end{abstract}

Subject headings: galaxies: abundances — galaxies: evolution — intergalactic medium

\section{INTRODUCTION}

We present an analytical and phenomenological model for the chemical evolution of galaxies based on hierarchical structure formation. This model provides a possible explanation for the metal abundances observed in damped Ly $\alpha$ (DLA) systems and the metallicity distribution of stars in galaxies. It also suggests a possible mechanism for the formation of globular clusters. Measurement of "metallicities" of DLA systems by Lu et al. (1996) and further studies by Prochaska \& Wolfe $(2000,2002)$ showed that these systems have a large dispersion in $[\mathrm{Fe} / \mathrm{H}] \equiv \log (\mathrm{Fe} / \mathrm{H})-\log (\mathrm{Fe} / \mathrm{H})_{\odot}$ for a given redshift $z$ and that there appears to be a baseline enrichment at the level of $[\mathrm{Fe} / \mathrm{H}] \sim-3$ over a wide range of redshift, $1.5 \lesssim z \lesssim 4.5$. The work by Prochaska et al. (2003) provided a much more extensive database over a wider range of redshift, $0.5<z<5$. All these data exhibited the same behavior. We earlier found that the data on DLA systems could be explained by a simple closed-system model (Wasserburg \& Qian 2000b; Fig. 1 in this paper), in which the number abundance $(\mathrm{Fe} / \mathrm{H})$ of Fe relative to $\mathrm{H}$ atoms in a DLA system is determined by

$$
(\mathrm{Fe} / \mathrm{H})=(\mathrm{Fe} / \mathrm{H})_{P}+\left[P_{\mathrm{Fe}} /(\mathrm{H})\right]\left[t(z)-t^{*}\right]
$$

In the above equation, $(\mathrm{Fe} / \mathrm{H})_{P} \sim 10^{-3}(\mathrm{Fe} / \mathrm{H})_{\odot}\left([\mathrm{Fe} / \mathrm{H}]_{P} \sim\right.$ $-3)$ is the initial $\mathrm{Fe}$ abundance corresponding to a postulated prompt inventory of metals in the intergalactic medium

\footnotetext{
${ }^{1}$ School of Physics and Astronomy, University of Minnesota, Minneapolis, MN 55455; qian@physics.umn.edu.

2 The Lunatic Asylum, Division of Geological and Planetary Sciences, California Institute of Technology, Pasadena, CA 91125.
}

(IGM), $P_{\mathrm{Fe}} /(\mathrm{H})$ is the rate of Fe production by Type II supernovae (SNe II) per $\mathrm{H}$ atom in the gas of the DLA system and is taken to be $\sim(\mathrm{Fe} / \mathrm{H})_{\odot} /(30 \mathrm{Gyr})$ based on estimates for the Galaxy (see $\S 2), t(z)$ is the age of the universe at the redshift $z$ of the system, and $t^{*}$ is the turn-on time corresponding to the onset of astration and $\mathrm{Fe}$ production in the system. It was found that the available data on $[\mathrm{Fe} / \mathrm{H}]$ for essentially all the DLA systems lie between the prompt inventory value and the upper bound obtained for $t^{*}=0$. The large dispersion in $[\mathrm{Fe} / \mathrm{H}]$ at a given $z$ could be explained by different $t^{*}$ for different DLA systems. It was found that for the above closed-system model, most systems at a given $z$ have $t^{*}$ in a range close to $t(z)$ (i.e., they turned on long after the big bang so that their baryonic matter had to be stored in the IGM for this long period).

The astrophysical implications of the late turn-on of most DLA systems in the above closed-system model were explored by us in a recent study based on the cold dark matter (CDM) model of hierarchical structure formation (Qian \& Wasserburg 2003b). We there associated the evolution of a DLA system with the growth of a halo through infall of CDM and gas. The late turn-on was reinterpreted in terms of two factors. First, a halo must achieve some threshold mass before star formation can occur. As a halo associated with a low- $\sigma$ density fluctuation reaches a given mass at a later time, $\mathrm{Fe}$ production in such a halo would be delayed simply because of the later onset of star formation. Second, subsequent to the onset of star formation, the growth of $(\mathrm{Fe} / \mathrm{H})$ in the gas of a halo is determined by the competition between production by $\mathrm{SNe}$ II and dilution by gas infall. The uniform growth with time does not set in until infall ceases. Thus, the late turn-on does not require the introduction of an arbitrary delay time $t^{*}$ but is a direct result of the infall rates in the CDM model of hierarchical structure formation. 


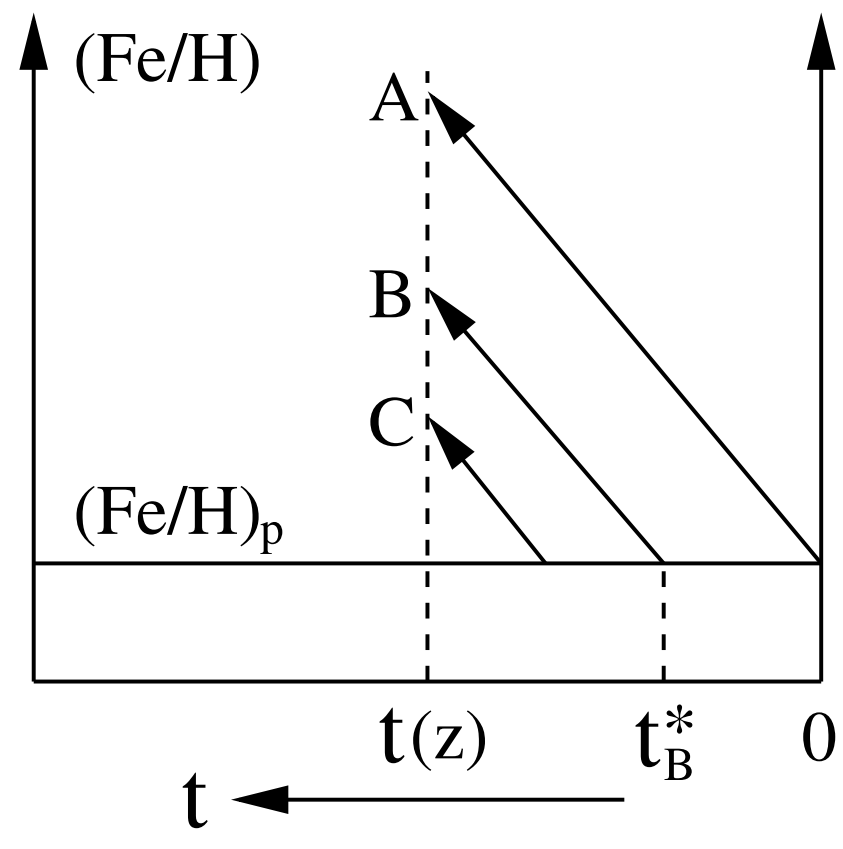

FIG. 1.- Schematic diagram of the closed-system model for $(\mathrm{Fe} / \mathrm{H})$ in DLA systems. The baseline $(\mathrm{Fe} / \mathrm{H})_{P}$ is the value corresponding to a postulated prompt inventory in the IGM. Normal astration and Fe production by SNe II start in a DLA system at time $t^{*}$ after the big bang $(t=0)$. Different $t^{*}$ values for different DLA systems result in a wide range of $(\mathrm{Fe} / \mathrm{H})$ at a fixed $z$. For $t^{*}=0$, there is a maximum increase in $(\mathrm{Fe} / \mathrm{H})($ trajectory $\mathrm{A})$.

We here extend our model for the chemical evolution of DLA systems to a more general discussion of galactic chemical evolution. In $\S 2$ we review the consequences of astration concurrent with infall for the chemical evolution of gas in a halo that is not disrupted by SNe II. We discuss the effects of infall cessation, particularly the resulting metallicity distribution of stars in $\S 3$. The connection with the CDM model of hierarchical structure formation is described in $\S 4$. A comparison of our chemical evolution model with the data on DLA systems is presented in $\S 5$. Possible scenarios of globular cluster formation in connection with this model are discussed and conclusions given in $\S 6$.

\section{CHEMICAL EVOLUTION OF GAS IN AN ACCRETING HALO WITHOUT DISRUPTION}

We first consider the chemical evolution of gas in a halo that grows according to hierarchical structure formation. We assume that CDM and gas are fed into the halo at a fixed mass ratio through infall. For simplicity, the infalling gas from the IGM is assumed to be pure big bang debris (i.e., metal-free). Observations show that the IGM is significantly enriched, with metallicities corresponding to $[\mathrm{Fe} / \mathrm{H}] \sim-3$ over a wide range of redshift, $1.5 \leq z \leq 5.5$ (e.g., Songaila 2001; Pettini et al. 2003; see also Simcoe et al. 2002, 2004; Schaye et al. 2003; Aguirre et al. 2004). Thus, the assumption of metal-free infalling gas is not necessarily valid. However, possible metallicities at the level of $[\mathrm{Fe} / \mathrm{H}] \sim-3$ for the infalling gas are soon overwhelmed by enrichments associated with astration in the halo. Insofar as the evolution of the halo substantially enhances the metal content of its gas to well above $[\mathrm{Fe} / \mathrm{H}] \sim-3$, neglecting the metallicity of the infalling gas is justified.

The history of star formation in the halo is crucial to the chemical evolution of the gas. The issue of what the first generation of stars were like has long been a matter of intense interest. Numerical simulations of collapse and fragmentation of metal-free gas clouds indicate that the first stars (or baryonic aggregates) were very massive. Formation of normal stars may not have occurred until a metallicity corresponding to $[\mathrm{Fe} / \mathrm{H}] \sim-4$ was reached in the IGM (see Bromm \& Larson 2004 and references therein). We do not consider the effect of this threshold metallicity that may possibly be required for normal astration. We assume that normal astration proceeds at a constant rate per $\mathrm{H}$ atom in the gas once the halo reaches some critical mass (see $\S 4$ ). From the discovery of a low-mass Galactic halo star with $[\mathrm{Fe} / \mathrm{H}]=-5.3$ (Christlieb et al. 2002), it is evident that some low-mass stars were formed when the level of heavy metals in the IGM was extremely low, far below the threshold metallicity for normal astration suggested by theoretical models (Bromm et al. 2001).

It has long been recognized that stars in the Galactic halo form a distinct population. Extensive observational work has established that these stars have distinctively low $[\mathrm{Fe} / \mathrm{H}]$, ranging from typically approximately -2 (e.g., Carney et al. 1996) down to -5.3 (see Fig. 5 in Christlieb 2003). There is a clear break at $[\mathrm{Fe} / \mathrm{H}] \sim-1$ observed in the Galactic evolution of $[\mathrm{Ba} / \mathrm{Fe}]$ and $[\mathrm{O} / \mathrm{Fe}]$ with $[\mathrm{Fe} / \mathrm{H}]$. For $[\mathrm{Ba} / \mathrm{Fe}]$, this break is due to the lack of major $s$-process contributions from asymptotic giant branch stars to the $\mathrm{Ba}$ in the interstellar medium (ISM) for $[\mathrm{Fe} / \mathrm{H}]<-1$ (e.g., Busso et al. 1999), while for $[\mathrm{O} / \mathrm{Fe}]$, it is due to the lack of major Fe contributions from SNe Ia for $[\mathrm{Fe} / \mathrm{H}]<-1$ (Tinsley 1979; see also Kobayashi et al. 1998). In addition, the abundance patterns of the elements from the Fe group down to $\mathrm{O}$ observed in Galactic halo stars are characteristic of the nucleosynthetic yields of SNe II. Thus, the overall abundances of metals in the early Galaxy corresponding to $[\mathrm{Fe} / \mathrm{H}]<-1$ in the ISM were clearly dominated by contributions from SNe II with massive $(\sim 10-100$ $\left.M_{\odot}\right)$ progenitors. We note that the marked change at $[\mathrm{Fe} / \mathrm{H}] \sim-3$ observed for the abundances of heavy $r$-process nuclei in Galactic halo stars (e.g., McWilliam et al. 1995; Sneden et al. 1996; Westin et al. 2000; Burris et al. 2000) appears to require a source other than SNe II for these nuclei (Qian \& Wasserburg 2003a). However, this $r$-process source cannot produce any significant amount of the elements from the Fe group down to O (e.g., Qian \& Wasserburg 2002) and therefore does not affect our discussion of the chemical evolution of these elements here.

Based on the above discussion, we only consider metal production by $\mathrm{SNe}$ II subsequent to the onset of astration during the early evolution of a halo. The general effects of $\mathrm{SNe} \mathrm{Ia}$, which mainly contribute the Fe group elements at $[\mathrm{Fe} / \mathrm{H}] \gtrsim-1$, are discussed in $\S 3$. For convenience, we use $\mathrm{Fe}$ as a representative metal element. At $[\mathrm{Fe} / \mathrm{H}]<-1$, the other elements (from the Fe group down to $\mathrm{O}$ ) essentially evolve in proportion to $\mathrm{Fe}$ as specified by the effective yield pattern of SNe II (see Table 1 in Qian \& Wasserburg 2002). If gas in the halo is homogeneous and is not expelled by explosions of $\mathrm{SNe}$ II, the basic equation governing its chemical evolution (Qian \& Wasserburg 2003b) can be written as

$$
\frac{d(\mathrm{Fe} / \mathrm{H})}{d t}=\frac{P_{\mathrm{Fe}}}{(\mathrm{H})}-\frac{1}{(\mathrm{H})} \frac{d(\mathrm{H})_{\mathrm{in}}}{d t}(\mathrm{Fe} / \mathrm{H}) .
$$

Here $(\mathrm{Fe} / \mathrm{H})$ is the number ratio of $\mathrm{Fe}$ to $\mathrm{H}$ atoms in the gas, $P_{\mathrm{Fe}}$ is the net rate of $\mathrm{Fe}$ production by $\mathrm{SNe} \mathrm{II},(\mathrm{H})$ is the number of $\mathrm{H}$ atoms in the gas, and $d(\mathrm{H})_{\text {in }} / d t$ is the rate of 


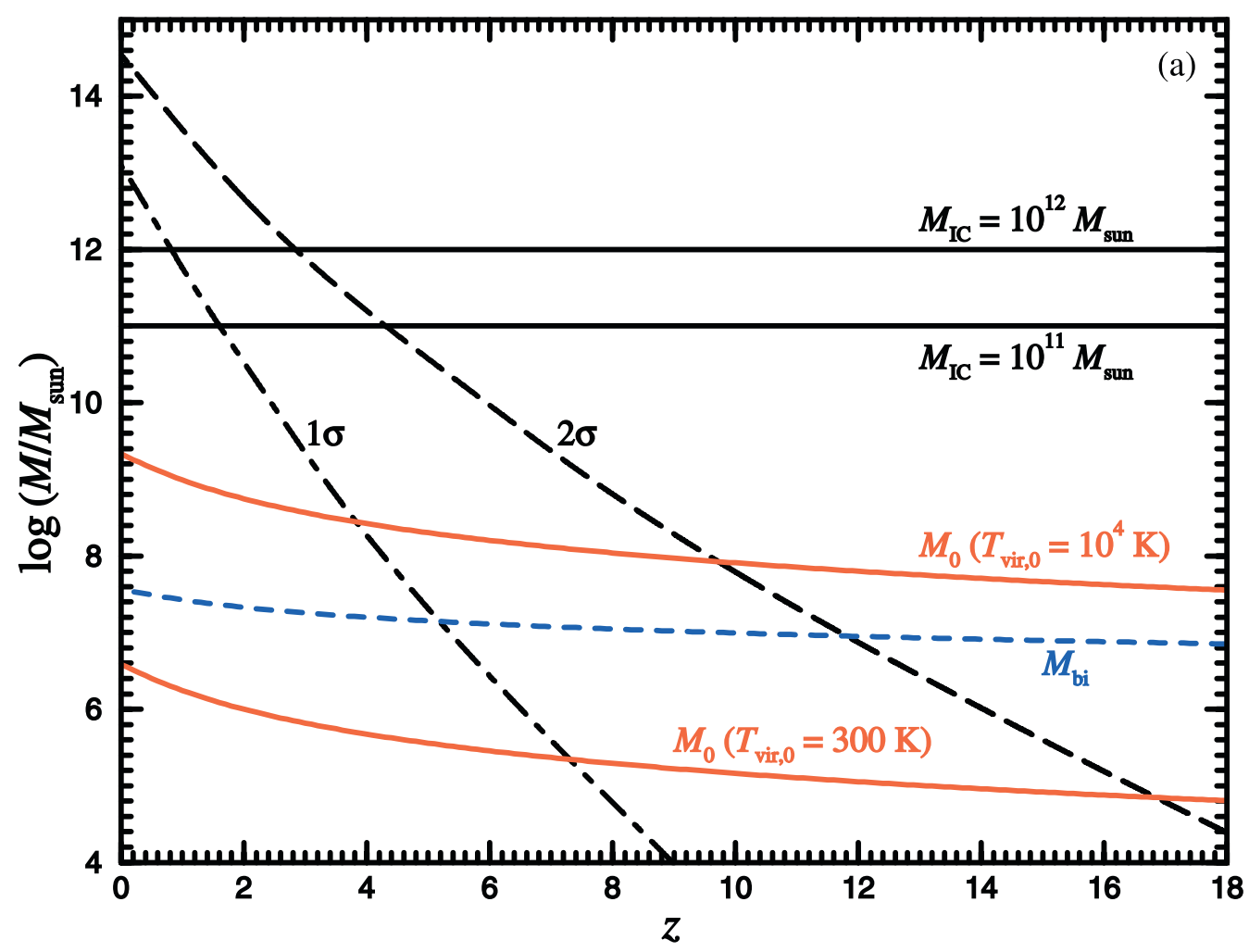

FIG. 2.-(a) Evolution of the halo mass $M$ as a function of $z$ calculated for $1 \sigma$ (dot-dashed curve) and $2 \sigma$ (long-dashed curve) halos using the model of hierarchical structure formation discussed in Barkana \& Loeb (2001). For a specific halo, this evolution determines the infall rate. A simple estimate of the infall rate for 1 and $2 \sigma$ halos can be obtained by taking a typical slope of $|d \ln M / d z| \sim 2.3$. It is assumed that infall ceases when $M$ reaches $M_{\mathrm{IC}}$. For $M_{\mathrm{IC}}=10^{11} M_{\odot}$ (horizontal solid line labeled " $M_{\mathrm{IC}}=10^{11} M_{\odot}$ "), this occurs at redshifts $z_{\mathrm{IC}}=1.6$ and 4.3 for 1 and $2 \sigma$ halos, respectively, while for $M_{\mathrm{IC}}=10^{12} M_{\odot}$ (horizontal solid line labeled " $M_{\mathrm{IC}}=10^{12} M_{\odot}$ "), this occurs at redshifts $z_{\mathrm{IC}}=0.8$ and 2.8 for 1 and $2 \sigma$ halos, respectively. The time at which the virial temperature of the gas reaches a critical value $T_{\mathrm{vir}, 0}$ is taken as the onset of astration in a halo. The halo mass $M_{0}$ required to reach $T_{\mathrm{vir}, 0}$ is shown as a function of $z$ for $T_{\mathrm{vir}, 0}=300 \mathrm{~K}$ [solid curve labeled " $M_{0}\left(T_{\mathrm{vir}, 0}=300 \mathrm{~K}\right)$ "] and $T_{\mathrm{vir}, 0}=10^{4} \mathrm{~K}$ [solid curve labeled " $M_{0}\left(T_{\mathrm{vir}, 0}=10^{4} \mathrm{~K}\right)$ "], respectively. The redshift $z_{0}$ at which the curve $M_{0}(z)$ intersects the curve $M(z)$ for a halo corresponds to the onset of astration. For $T_{\mathrm{vir}, 0}=300 \mathrm{~K}, z_{0}=7.3$ and 16.8 for 1 and $2 \sigma$ halos, respectively, while for $T_{\mathrm{vir}, 0}=10^{4} \mathrm{~K}$, $z_{0}=3.8$ and 9.7 for 1 and $2 \sigma$ halos, respectively. It is assumed that a single SN II can expel all the gas from a halo before the binding energy of the gas $E_{\mathrm{bi}, \text { gas }}$ reaches the explosion energy of $10^{51} \mathrm{ergs}$ for an SN II. The halo mass $M_{\mathrm{bi}}$ required to reach $E_{\mathrm{bi}, \text { gas }}=10^{51}$ ergs is shown as a function of $z$ (short-dashed curve labeled " $M_{\mathrm{bi}}$ "). (b) Evolution of [Fe/ $\left.\mathrm{H}\right]$ for $1 \sigma$ (dot-dashed curve) and $2 \sigma$ (long-dashed curve) halos as functions of $z$. For both halos, it is assumed that the onset of astration corresponds to $T_{\mathrm{vir}, 0}=10^{4} \mathrm{~K}$, instant infall cessation occurs for $M_{\mathrm{IC}}=10^{11} M_{\odot}$, and SNe Ia start to contribute Fe at [Fe/H] $=-1$. The solid curve labeled "no infall" is for a system with no infall. For all cases, the Fe production rate per $\mathrm{H}$ atom of the gas is taken to be $\lambda_{\mathrm{Fe}}=(30 \mathrm{Gyr})^{-1}$ when only SNe II provide $\mathrm{Fe}([\mathrm{Fe} / \mathrm{H}]<-1)$ and $3 \lambda_{\mathrm{Fe}}$ when both SNe II and Ia provide $\mathrm{Fe}([\mathrm{Fe} / \mathrm{H}]>-1)$. Using the simple estimate of the infall rate, the case in which the Fe production by SNe II only is balanced by the dilution due to infall is shown as the solid curve labeled "quasi-steady state estimate." The overall evolution of [Fe/H] for a $2 \sigma$ halo is indicated by arrows. Note the sharp initial rise of the evolutionary trajectories for both 1 and $2 \sigma$ halos subsequent to the onset of astration. These trajectories then lie close to the QSS estimate until infall cessation $\left(z_{\mathrm{IC}}\right)$. They rapidly approach the case of no infall after infall cessation $\left(z<z_{\mathrm{IC}}\right)$. (c) Effects of $T_{\mathrm{vir}, 0}$ and $M_{\mathrm{IC}}$ on the evolution of $[\mathrm{Fe} / \mathrm{H}]$ for a $2 \sigma$ halo. The solid curve is the case shown in $(b)$. The dot-dashed curve assumes a different $T_{\mathrm{vir}, 0}$ of $300 \mathrm{~K}$, and the short-dashed curve assumes a different $M_{\mathrm{IC}}$ of $10^{12} M_{\odot}$. Note that these three cases share a common period of QSS evolution.

infall of $\mathrm{H}$ atoms into the halo. The time $t=0$ corresponds to the big bang. Note that the formal integration of equation (2) gives a result similar to equation (1) with the integral of the infall term playing the role of the delay time $t^{*}$. The equation governing $(\mathrm{H})$ is

$$
\frac{d(\mathrm{H})}{d t}=\frac{d(\mathrm{H})_{\mathrm{as}}}{d t}+\frac{d(\mathrm{H})_{\mathrm{in}}}{d t},
$$

where $d(\mathrm{H})_{\text {as }} / d t<0$ is the astration rate. During the infalldominated regime, $\left|d(\mathrm{H})_{\text {as }} / d t\right| \ll d(\mathrm{H})_{\text {in }} / d t$, so that $(\mathrm{H}) \approx$ $(\mathrm{H})_{\text {in }}$. Then, for this regime equation (2) can be rewritten as

$$
\frac{d Z_{\mathrm{Fe}}}{d t}=\lambda_{\mathrm{Fe}}-\lambda_{\mathrm{in}} Z_{\mathrm{Fe}}
$$

where $Z_{\mathrm{Fe}} \equiv(\mathrm{Fe} / \mathrm{H}) /(\mathrm{Fe} / \mathrm{H})_{\odot}, \lambda_{\mathrm{Fe}} \equiv P_{\mathrm{Fe}} /\left[(\mathrm{Fe} / \mathrm{H})_{\odot}(\mathrm{H})\right]$, and $\lambda_{\text {in }} \equiv d \ln (\mathrm{H})_{\text {in }} / d t$. Note that for $Z_{\mathrm{Fe}}$ to increase rapidly, $\lambda_{\text {in }} Z_{\mathrm{Fe}}$ must be significantly smaller than $\lambda_{\mathrm{Fe}}$.
The general solution to equation (4) for a specific halo is

$$
Z_{\mathrm{Fe}}(t)=\exp \left[-\int_{t_{0}}^{t} \lambda_{\mathrm{in}}\left(t^{\prime}\right) d t^{\prime}\right] \int_{t_{0}}^{t} \lambda_{\mathrm{Fe}}\left(t^{\prime \prime}\right) \exp \left[\int_{t_{0}}^{t^{\prime \prime}} \lambda_{\mathrm{in}}\left(t^{\prime \prime \prime}\right) d t^{\prime \prime \prime}\right] d t^{\prime \prime},
$$

where $t_{0}$ is the onset of astration and Fe production [i.e., $Z_{\mathrm{Fe}}(t) \equiv 0$ for $\left.t \leq t_{0}\right]$. The time $t$ can be related to the mass of the halo at that time based on the density fluctuation associated with the halo (see Fig. $2 a ; \S 4$ ). Given the rate of infall $\lambda_{\text {in }}$ and the rate of $\mathrm{Fe}$ production per $\mathrm{H}$ atom in the gas $\lambda_{\mathrm{Fe}}, Z_{\mathrm{Fe}}(t)$ only depends on the time $t_{0}$ for onset of astration and the time $t$. For the infall phase, we explicitly assume that $P_{\mathrm{Fe}} /(\mathrm{H})$ is constant and take $\lambda_{\mathrm{Fe}}=(30 \mathrm{Gyr})^{-1}$. This value of $\lambda_{\mathrm{Fe}}$ corresponds to the average rate of Fe production by $\mathrm{SNe}$ II in the Galaxy $[\sim 1 / 3$ of $(\mathrm{Fe} / \mathrm{H}) \odot$ over a period of $\sim 10 \mathrm{Gyr}]$. The infall rate $\lambda_{\text {in }}$ is not an arbitrary function of $t$ but is directly determined from the model of hierarchical structure formation 


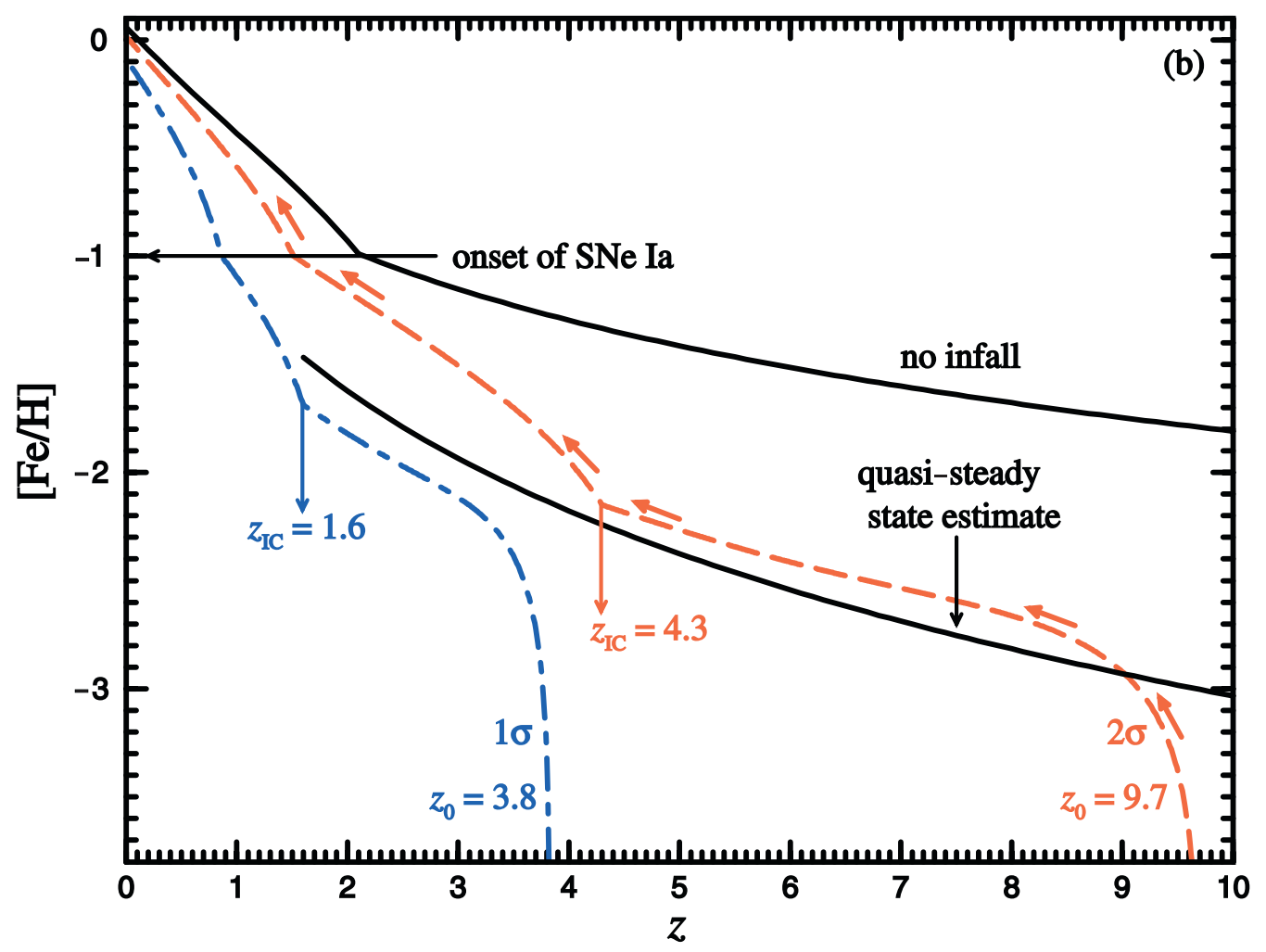

FIG. 2.-Continued

discussed in Barkana \& Loeb (2001) (see below). The evolution of $[\mathrm{Fe} / \mathrm{H}]$ in halos with the infall of big bang debris but without gas expulsion by $\mathrm{SNe}$ II can then be calculated in a straightforward manner.

The rate of gas infall governs the growth of the baryonic mass of a halo. We assume that a constant fraction $\Omega_{b} / \Omega_{m}$ of the total halo mass is in baryonic matter, where $\Omega_{b}$ and $\Omega_{m}$ are the fractions of the present critical density contributed by baryonic and all matter, respectively. The infall rate is then related to the total halo mass $M$ (mostly in CDM) as

$$
\lambda_{\text {in }}(t)=\frac{d \ln M}{d t}=\frac{d \ln M}{d z} \frac{d z}{d t} .
$$

For a flat universe, we have

$$
\frac{d z}{d t}=-H_{0}(1+z) \sqrt{\Omega_{m}(1+z)^{3}+\Omega_{\Lambda}},
$$

where $H_{0}=100 \mathrm{hm} \mathrm{s}^{-1} \mathrm{Mpc}^{-1}$ is the present Hubble parameter and $\Omega_{\Lambda}$ is the fraction of the present critical density contributed by the cosmological constant. We take $h=0.7$, $\Omega_{b}=0.045, \Omega_{m}=0.3$, and $\Omega_{\Lambda}=0.7$. Based on the model of hierarchical structure formation (see $\S 4$ ), we show $M$ as a function of $z$ for halos associated with 1 and $2 \sigma$ density fluctuations (1 and $2 \sigma$ halos), respectively, in Figure $2 a$. Using the function $M(z)$ for a specific halo, we calculate $\lambda_{\text {in }}(t)$ from equations (6) and (7). Assuming that astration starts (see Fig. $2 a ; \S 4)$ when a $1 \sigma$ halo reaches $M\left(z_{0}\right)=2.8 \times 10^{8} M_{\odot}$, corresponding to $z_{0}=3.8\left(t_{0}=1.6 \mathrm{Gyr}\right)$, or when a $2 \sigma$ halo reaches $M\left(z_{0}\right)=8.4 \times 10^{7} M_{\odot}$, corresponding to $z_{0}=9.7$ $\left(t_{0}=0.49 \mathrm{Gyr}\right)$, we numerically integrate equation (4) to calculate the evolution of $[\mathrm{Fe} / \mathrm{H}]=\log Z_{\mathrm{Fe}}$ as a function of $z$ until $z=1.6\left[t=4.1 \mathrm{Gyr}, M(z)=10^{11} M_{\odot}\right]$ for the $1 \sigma$ halo or until $z=4.3\left[t=1.4 \mathrm{Gyr}, M(z)=10^{11} M_{\odot}\right]$ for the $2 \sigma$ halo. The results are shown in Figure $2 b$. If we consider smaller values of $M\left(z_{0}\right)$, for instance, $M\left(z_{0}\right) \sim 10^{5} M_{\odot}$, then the onset of astration will be moved to higher redshift. The evolution of $[\mathrm{Fe} / \mathrm{H}]$ for a $2 \sigma$ halo with $M\left(z_{0}\right)=7.1 \times 10^{4} M_{\odot}$, corresponding to $z_{0}=16.8\left(t_{0}=0.23 \mathrm{Gyr}\right)$, is shown as the dot-dashed curve in Figure $2 c$, for which the case for $M\left(z_{0}\right)=$ $8.4 \times 10^{7} M_{\odot}$, corresponding to $z_{0}=9.7$, is also shown as the solid curve. It can be seen that the dot-dashed curve merges with the solid curve at $z \sim 8$. This is because [Fe/H] quickly approaches a quasi-steady state (QSS) subsequent to the onset of astration (see below).

Inspection of equation (4) suggests that if $\lambda_{\text {in }}(t) \gg$ $\left|d \ln Z_{\mathrm{Fe}}(t) / d t\right|$, then a QSS exists such that

$$
Z_{\mathrm{Fe}}(t) \approx Z_{\mathrm{Fe}}^{\mathrm{QSS}}(t) \equiv \lambda_{\mathrm{Fe}} / \lambda_{\mathrm{in}}(t)
$$

The approach of $Z_{\mathrm{Fe}}(t)$ to $Z_{\mathrm{Fe}}^{\mathrm{QSS}}(t)$ can be approximated as

$$
Z_{\mathrm{Fe}}(t) \approx Z_{\mathrm{Fe}}^{\mathrm{QSS}}(t)-Z_{\mathrm{Fe}}^{\mathrm{QSS}}\left(t_{0}\right) \exp \left[-\int_{t_{0}}^{t} \lambda_{\mathrm{in}}\left(t^{\prime}\right) d t^{\prime}\right] .
$$

For $t>t_{0}+\left[\lambda_{\text {in }}\left(t_{0}\right)\right]^{-1}$, the term containing the exponential in equation (9) is insignificant, and $Z_{\mathrm{Fe}}(t)$ approximately equals the QSS value $Z_{\mathrm{Fe}}^{\mathrm{QSS}}(t)$ (see Qian \& Wasserburg 2003b). When we numerically integrate equation (4) to calculate the evolution of $[\mathrm{Fe} / \mathrm{H}]=\log Z_{\mathrm{Fe}}$ as a function of $z$, we always use the actual function $M(z)$ for a specific halo to obtain the corresponding $\lambda_{\text {in }}(t)$. For a simple estimate of $\lambda_{\text {in }}(t)$, we note that the slopes of the $M(z)$ curves are $|d \ln M / d z| \sim 2.3$ within a factor of a few for both 1 and $2 \sigma$ halos (see Fig. $2 a$ ). We 


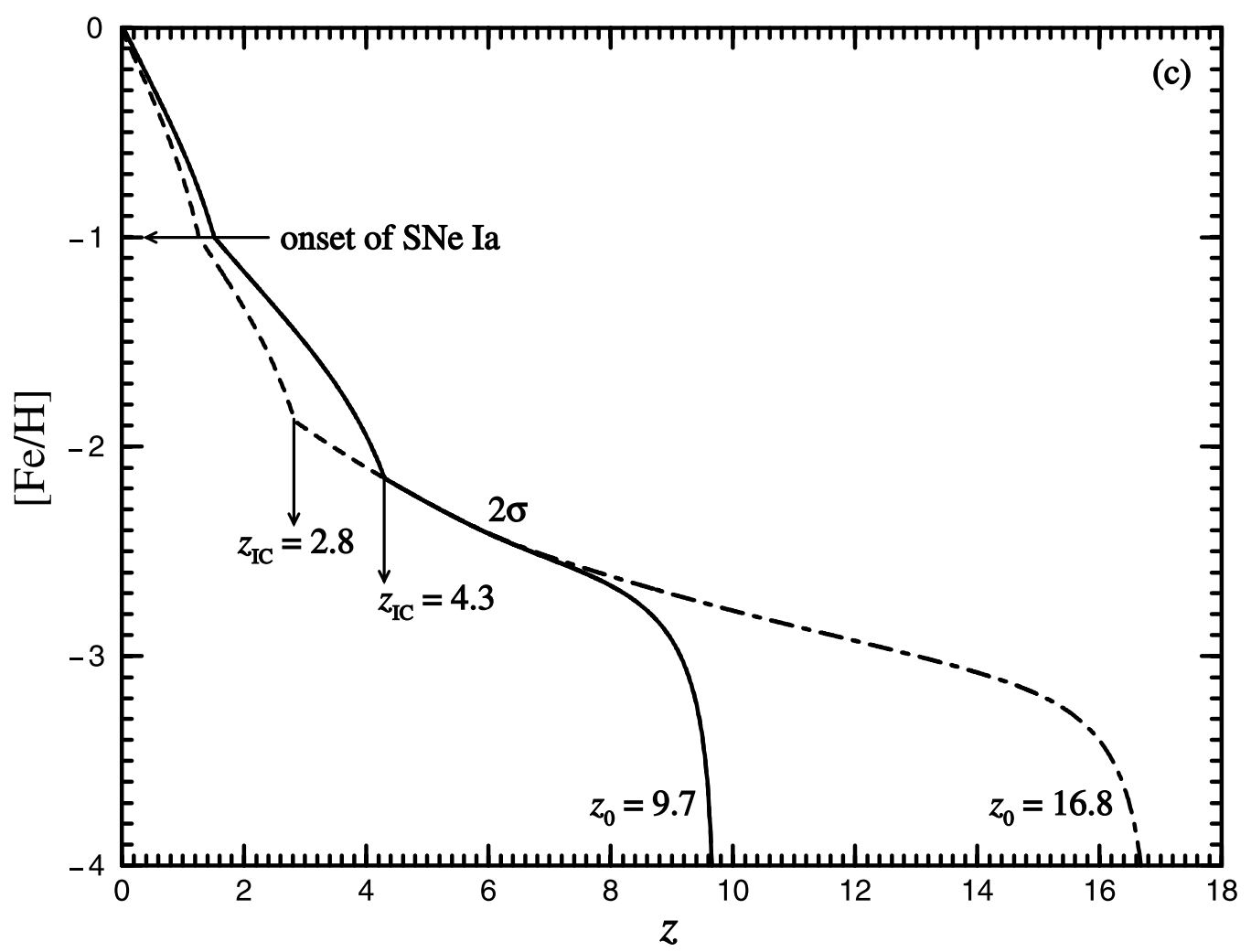

FIG. 2.-Continued

also note that for $z>1, \Omega_{\Lambda}$ can be ignored in equation (7) to give

$$
t \approx \frac{2}{3} \frac{1}{H_{0} \sqrt{\Omega_{m}(1+z)^{3}}} \approx 17(1+z)^{-3 / 2} \mathrm{Gyr} .
$$

Using $|d \ln M / d z| \sim 2.3$ and equations (6) and (10), we obtain

$$
\lambda_{\text {in }}(t) \sim 10(\mathrm{Gyr} / t)^{5 / 3} \mathrm{Gyr}^{-1} \sim 0.09(1+z)^{5 / 2} \mathrm{Gyr}^{-1}
$$

as a rough estimate for both 1 and $2 \sigma$ halos. This gives for these halos

$$
Z_{\mathrm{Fe}}^{\mathrm{QSS}}(t) \sim(1 / 300)(t / \mathrm{Gyr})^{5 / 3} \sim 0.4(1+z)^{-5 / 2},
$$

or

$$
[\mathrm{Fe} / \mathrm{H}]_{\mathrm{QSS}}=\log Z_{\mathrm{Fe}}^{\mathrm{QSS}}(t) \sim-2.9-2.5 \log [(1+z) / 10] .
$$

The above estimate for $[\mathrm{Fe} / \mathrm{H}]_{\mathrm{QSS}}$ is shown as the solid curve labeled "quasi-steady state estimate" in Figure $2 b$.

It can be seen from Figure $2 b$ that for both 1 and $2 \sigma$ halos, the $[\mathrm{Fe} / \mathrm{H}]$ of the gas initially rises very rapidly and approaches the QSS value, which ranges from $[\mathrm{Fe} / \mathrm{H}]_{\mathrm{QSS}} \sim$ -2.8 at $z=8$ to -1.6 at $z=2$. For comparison, the case of evolution from an initial metal-free state without infall, $Z_{\mathrm{Fe}}(t)=\lambda_{\mathrm{Fe}} t$ (prior to the onset of $\mathrm{SNe}$ Ia), is also shown in Figure $2 b$. This should give an upper bound to $Z_{\mathrm{Fe}}$ for all halos and appears to be a reasonable bound for the observational data on DLA systems (Wasserburg \& Qian 2000b; Qian \& Wasserburg 2003b; see Fig. 5 in this paper). Compared to the case of no infall, the Fe enrichment for 1 and $2 \sigma$ halos is suppressed because of dilution by infall by a factor of $\sim \lambda_{\text {in }}(t) t \sim 10(\mathrm{Gyr} / t)^{2 / 3}$, which ranges from a factor of $\sim 4.5$ for $t=3.3$ Gyr $(z=2)$ to a factor of $\sim 14$ for $t=0.63 \mathrm{Gyr}$ $(z=8)$.

\section{EFFECTS OF INFALL CESSATION}

We now consider that the infall of gas ceases at some time for a halo and investigate the subsequent chemical evolution of gas in the halo. The metallicity distribution of stars formed before and after infall cessation (IC) is studied. The general effects of $\mathrm{SNe}$ Ia on the chemical evolution of gas and stars in a halo are also discussed at the end of this section.

\subsection{Chemical Evolution of Gas}

We first consider the chemical evolution of gas in a halo after continuous infall ceases sharply at some time $t_{\mathrm{IC}}$. The governing equations in this case are still equations (2)-(4), but now $d(\mathrm{H})_{\text {in }} / d t=0, \lambda_{\text {in }}=0$, and the new initial state has $Z_{\mathrm{Fe}}\left(t_{\mathrm{IC}}\right) \approx Z_{\mathrm{Fe}}^{\mathrm{QSS}}\left(t_{\mathrm{IC}}\right)$ as determined by the prior evolution with infall. Thus, for $t>t_{\mathrm{IC}}$ but prior to the onset of $\mathrm{SNe}$ Ia (see $\S 3.3)$,

$$
Z_{\mathrm{Fe}}(t)=Z_{\mathrm{Fe}}\left(t_{\mathrm{IC}}\right)+\lambda_{\mathrm{Fe}}\left(t-t_{\mathrm{IC}}\right)
$$

Taking $t_{\mathrm{IC}}=4.1$ Gyr $\left(z_{\mathrm{IC}}=1.6\right)$ for a $1 \sigma$ halo and $t_{\mathrm{IC}}=$ $1.4 \mathrm{Gyr}\left(z_{\mathrm{IC}}=4.3\right)$ for a $2 \sigma$ halo, we show the above evolution of $[\mathrm{Fe} / \mathrm{H}]=\log Z_{\mathrm{Fe}}$ for these two halos in Figure $2 b$. It can be seen that the evolution subsequent to infall cessation departs markedly from the QSS case. For the $2 \sigma$ halo, the evolution clearly begins to approach the case of no infall. In these calculations, we have assumed that infall ceases sharply at time $t_{\mathrm{IC}}$ corresponding to redshift $z_{\text {IC }}$ when the mass of a halo reaches $M\left(z_{\text {IC }}\right)=10^{11} M_{\odot}($ see Fig. $2 a ; \S 4)$. If we consider larger 
values of $M\left(z_{\mathrm{IC}}\right)$, for instance, $M\left(z_{\mathrm{IC}}\right)=10^{12} M_{\odot}$, this will only delay the onset of uniform growth of $Z_{\mathrm{Fe}}$ to lower redshift. The general evolution of $[\mathrm{Fe} / \mathrm{H}]$ outlined above will be unaffected. The evolution of $[\mathrm{Fe} / \mathrm{H}]$ for a $2 \sigma$ halo with $M\left(z_{\mathrm{IC}}\right)=10^{12} M_{\odot}$, corresponding to $t_{\mathrm{IC}}=2.3 \mathrm{Gyr}\left(z_{\mathrm{IC}}=2.8\right)$, is shown as the dashed curve in Figure $2 c$.

\subsection{Metallicity Distribution of Stars}

In addition to the effect on the evolution of $Z_{\mathrm{Fe}}$ discussed above, the instant cessation of infall has another consequence. During the infall phase, the amount of gas in a halo rapidly increases as $(\mathrm{H}) \approx(\mathrm{H})_{\text {in }}$ for $\left|d(\mathrm{H})_{\text {as }} / d t\right| \ll d(\mathrm{H})_{\text {in }} / d t$. Subsequent to instant infall cessation, $(\mathrm{H})$ steadily decreases because of astration. Thus, $(\mathrm{H})$ is maximal at time $t_{\mathrm{IC}}$. As the rate of star formation increases with $(\mathrm{H})$, this rate also reaches a maximum at time $t_{\mathrm{IC}}$.

The general history of star formation before and after infall cessation is reflected by the metallicity distribution of stars in a halo. This distribution is defined as the number $(N)$ of stars formed per unit interval of $[\mathrm{Fe} / \mathrm{H}]=\log Z_{\mathrm{Fe}}$,

$$
\frac{d N}{d[\mathrm{Fe} / \mathrm{H}]}=-(\ln 10) \frac{Z_{\mathrm{Fe}}}{(\mathrm{H})_{*}} \frac{d(\mathrm{H})_{\mathrm{as}} / d t}{d Z_{\mathrm{Fe}} / d t},
$$

where $(\mathrm{H})_{*}$ is the average number of $\mathrm{H}$ atoms in a star [note that $\left.d(\mathrm{H})_{\text {as }} / d t<0\right]$.

The metallicity distribution of stars formed at $t>t_{\mathrm{IC}}$ is simple to obtain. In this case, $d(\mathrm{H}) / d t=d(\mathrm{H})_{\text {as }} / d t$ and $d Z_{\mathrm{Fe}} / d t=P_{\mathrm{Fe}} /\left[(\mathrm{Fe} / \mathrm{H})_{\odot}(\mathrm{H})\right]$ (see eqs. [3] and [4]). Assuming that the SN II rate is the same as the rate at which massive SN II progenitors are formed (the instantaneous recycling approximation), we can relate $P_{\mathrm{Fe}}$ to the astration rate $d(\mathrm{H})_{\mathrm{as}} / d t$ as

$$
P_{\mathrm{Fe}}=-\alpha(\mathrm{Fe} / \mathrm{H})_{\odot}\left[d(\mathrm{H})_{\mathrm{as}} / d t\right],
$$

where $\alpha$ is a positive dimensionless constant. Thus, for $t>t_{\mathrm{IC}}$,

$$
\frac{d Z_{\mathrm{Fe}}}{d t}=-\frac{\alpha}{(\mathrm{H})} \frac{d(\mathrm{H})_{\mathrm{as}}}{d t}=-\alpha \frac{d \ln (\mathrm{H})}{d t} .
$$

The above equation can be solved to give

$$
(\mathrm{H})=(\mathrm{H})_{\mathrm{IC}} \exp \left[-\frac{Z_{\mathrm{Fe}}-Z_{\mathrm{Fe}}\left(t_{\mathrm{IC}}\right)}{\alpha}\right],
$$

where $(\mathrm{H})_{\mathrm{IC}}$ is the number of $\mathrm{H}$ atoms in the gas at $t=t_{\mathrm{IC}}$. The metallicity distribution at $t>t_{\mathrm{IC}}$ is then

$$
\begin{aligned}
\frac{d N}{d[\mathrm{Fe} / \mathrm{H}]} & =-(\ln 10) \frac{Z_{\mathrm{Fe}}}{(\mathrm{H})_{*}} \frac{d(\mathrm{H})}{d Z_{\mathrm{Fe}}} \\
& =\frac{(\ln 10)}{\alpha} \frac{(\mathrm{H})_{\mathrm{IC}}}{(\mathrm{H})_{*}} Z_{\mathrm{Fe}} \exp \left[-\frac{Z_{\mathrm{Fe}}-Z_{\mathrm{Fe}}\left(t_{\mathrm{IC}}\right)}{\alpha}\right] .
\end{aligned}
$$

The above metallicity distribution assumes that only SNe II provide $\mathrm{Fe}$. Note that this distribution does not depend on the specific forms of $d(\mathrm{H})_{\mathrm{as}} / d t$ and $P_{\mathrm{Fe}}$ as functions of time but only on the ratio of these two quantities. This is true of any closed system as recognized by Hartwick (1976) long ago.

In contrast, the metallicity distribution of stars formed during the infall phase depends on the specific forms of
$d(\mathrm{H})_{\mathrm{as}} / d t$ and $P_{\mathrm{Fe}}$. In general, the evolution of $Z_{\mathrm{Fe}}$ and $(\mathrm{H})$ during the infall phase must be solved together as explicit functions of time from equations (2) and (3) after $d(\mathrm{H})_{\text {as }} / d t$, $P_{\mathrm{Fe}}$, and the infall rate $d(\mathrm{H})_{\text {in }} / d t$ are specified for a given halo. We again assume

$$
P_{\mathrm{Fe}} /\left[(\mathrm{Fe} / \mathrm{H})_{\odot}(\mathrm{H})\right]=\lambda_{\mathrm{Fe}}=(30 \mathrm{Gyr})^{-1},
$$

as in $\S 2$. Using this assumption and equation (16) gives

$$
\frac{d(\mathrm{H})_{\mathrm{as}}}{d t}=-\frac{\lambda_{\mathrm{Fe}}}{\alpha}(\mathrm{H}) .
$$

As a numerical example of estimating $\alpha$, we assume that $\mathrm{SNe}$ II with progenitors of $\sim 20-25 M_{\odot}$ produce $\sim 0.1 M_{\odot}$ of Fe, as in the case of SN 1987A, but no other SNe II produce Fe. For a Salpeter initial mass function $d N / d M_{*} \propto M_{*}^{-2.35}$ over a range of stellar masses $M_{*} \sim 0.1-100 M_{\odot}$, a total of $\sim 1700 M_{\odot}$ of stars are formed for each Fe-producing SN II. From equation (16) we obtain

$$
\alpha \sim \frac{0.1 M_{\odot}}{10^{-3}\left(1700 M_{\odot}\right)} \sim \frac{1}{17},
$$

where we have used an $\mathrm{Fe}$ mass fraction of $\sim 10^{-3}$ corresponding to the number abundance $(\mathrm{Fe} / \mathrm{H})_{\odot}$. Noting that the Fe yields of SNe II, especially the dependence of these yields on the progenitor masses, are rather uncertain, we take $\alpha=1 / 18$ as a nominal value and also examine the effects of a higher value of $\alpha=1 / 6$ below. For a halo with $10^{10} M_{\odot}$ of gas and $\lambda_{\mathrm{Fe}}=(30 \mathrm{Gyr})^{-1}$, the astration rate in equation (20) is 6 and $2 M_{\odot} \mathrm{yr}^{-1}$ for $\alpha=1 / 18$ and $1 / 6$, respectively. These astration rates are typically much smaller than the infall rate as assumed in $\S 2$. However, to be more accurate, we can solve $Z_{\mathrm{Fe}}$ and $(\mathrm{H})$ together as functions of time from equations (2), (3), and (20). These two functions can then be used to obtain the metallicity distribution at $t<t_{\mathrm{IC}}$ from equations (15) and (20). The metallicity distribution over the whole time domain $t<t_{\mathrm{IC}}$ and $t>t_{\mathrm{IC}}$ is shown for a $2 \sigma$ halo with $\alpha=1 / 18$, $t_{0}=0.49 \mathrm{Gyr}\left(z_{0}=9.7\right)$, and instant infall cessation at $t_{\mathrm{IC}}=1.4 \mathrm{Gyr}\left(z_{\mathrm{IC}}=4.3\right)$ in Figure $3 a$ (solid curve). It can be seen that this distribution has a sharp peak at $[\mathrm{Fe} / \mathrm{H}]=-2.2$ and a broad peak at $[\mathrm{Fe} / \mathrm{H}]=-1.3$. The broad peak occurs in the region $t>t_{\mathrm{IC}}$ and is easy to understand from equation (19). This equation shows that $d N / d[\mathrm{Fe} / \mathrm{H}]$ has a peak at $Z_{\mathrm{Fe}}=\alpha$, which corresponds to $[\mathrm{Fe} / \mathrm{H}]=\log \alpha=-1.3$ for $\alpha=1 / 18$.

The sharp peak in the metallicity distribution of stars occurs at $t=t_{\mathrm{IC}}$, the time of infall cessation. The origin of this peak can be understood by considering equation (15) in the immediate neighborhood of $t_{\mathrm{IC}}\left(t_{\mathrm{IC}}^{-}<t_{\mathrm{IC}}<t_{\mathrm{IC}}^{+}\right)$. Note that $d Z_{\mathrm{Fe}} / d t$ has a discontinuity at $t=t_{\mathrm{IC}}$, but $Z_{\mathrm{Fe}}$ and $d(\mathrm{H})_{\mathrm{as}} / d t$ are the same on both sides of this discontinuity. This gives

$R_{-/+} \equiv \frac{(d N / d[\mathrm{Fe} / \mathrm{H}])_{t_{\mathrm{IC}}^{-}}}{(d N / d[\mathrm{Fe} / \mathrm{H}])_{t_{\mathrm{IC}}^{+}}}=\frac{\left(d Z_{\mathrm{Fe}} / d t\right)_{t_{\mathrm{IC}}^{+}}}{\left(d Z_{\mathrm{Fe}} / d t\right)_{t_{\mathrm{IC}}^{-}}}=\frac{\lambda_{\mathrm{Fe}}}{\left(d Z_{\mathrm{Fe}} / d t\right)_{t_{\mathrm{IC}}^{-}}}$.

As the QSS approximation $Z_{\mathrm{Fe}} \approx Z_{\mathrm{Fe}}^{\mathrm{QSS}}$ holds during the infall phase $\left(t<t_{\mathrm{IC}}\right),\left(d Z_{\mathrm{Fe}} / d t\right)_{t_{\mathrm{IC}}^{-}} \approx 0$ to zeroth order and $R_{-/+}$ is singular (see eq. [4]). To first order,

$$
\left(\frac{d Z_{\mathrm{Fe}}}{d t}\right)_{t_{\mathrm{IC}}^{-}}=\left(\frac{d Z_{\mathrm{Fe}}^{\mathrm{QSS}}}{d t}\right)_{t_{\mathrm{IC}}}=\left[\frac{d\left(\lambda_{\mathrm{Fe}} / \lambda_{\mathrm{in}}\right)}{d t}\right]_{t_{\mathrm{IC}}} \sim \frac{\lambda_{\mathrm{Fe}}}{6}\left(\frac{t_{\mathrm{IC}}}{\mathrm{Gyr}}\right)^{2 / 3},
$$



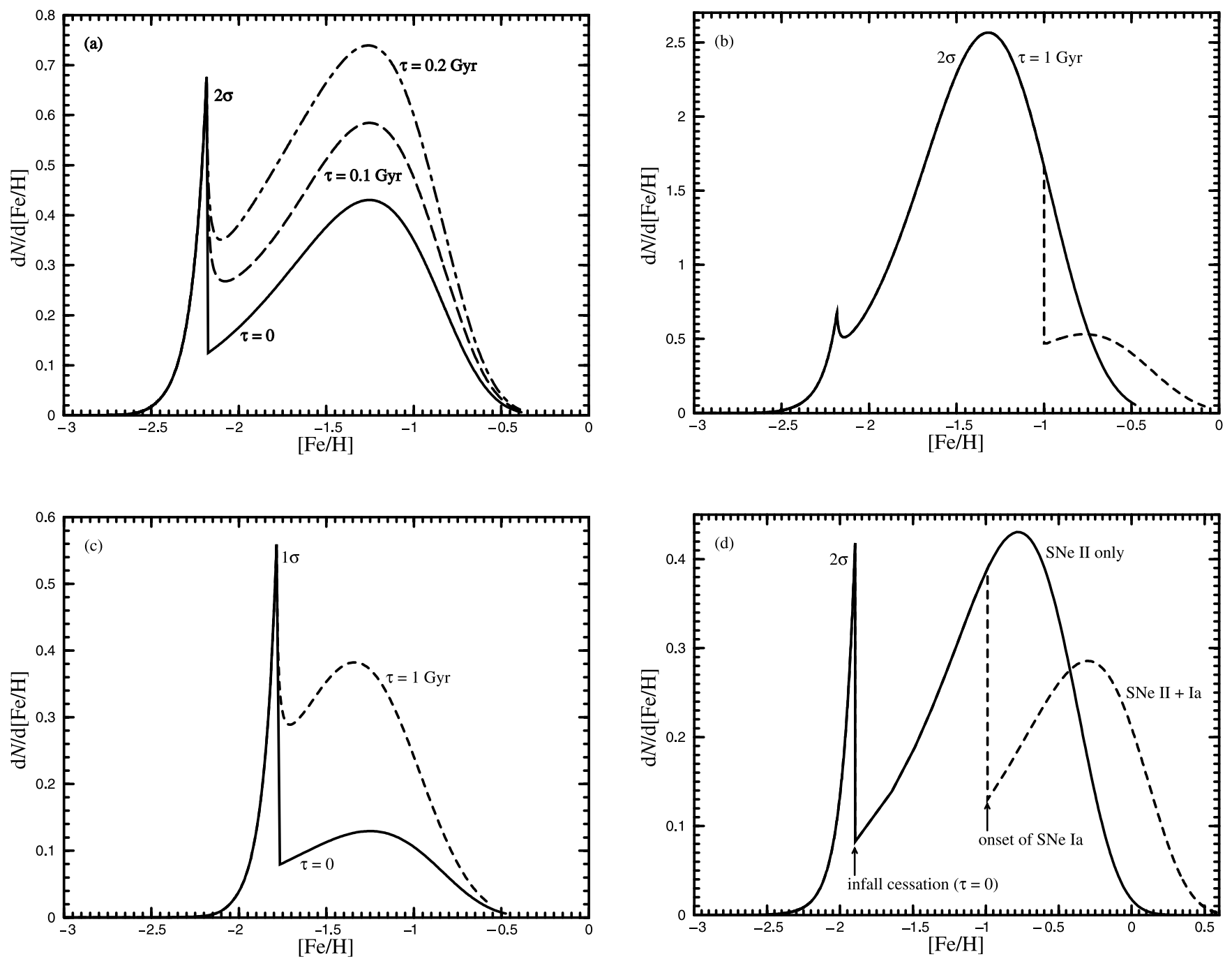

FIG. 3.-(a) Metallicity distribution (in arbitrary units) of stars in a $2 \sigma$ halo. It is assumed that astration starts at $z_{0}=9.7$, corresponding to $T_{\text {vir }, 0}=10^{4} \mathrm{~K}$, and infall starts to decrease exponentially on a timescale $\tau$ at $z_{\mathrm{IC}}=4.3$, corresponding to $M_{\mathrm{IC}}=10^{11} M_{\odot}$. Only SNe II provide Fe, at a rate of $\lambda_{\mathrm{Fe}}=(30 \mathrm{Gyr})^{-1}$ per $\mathrm{H}$ atom in the gas. The parameter $\alpha$ for the astration rate is taken to be $1 / 18$. The sharp peak in the metallicity distribution at $[\mathrm{Fe} / \mathrm{H}]=-2.2 \mathrm{represents}$ the stars formed during the infall phase $\left(z>z_{\mathrm{IC}}\right)$, while the broad peak at $[\mathrm{Fe} / \mathrm{H}]=\log \alpha=-1.3$ represents those formed subsequently $\left(z<z_{\mathrm{IC}}\right)$ until the gas is exhausted by astration. The rapid drop of the metallicity distribution at $z=z_{\mathrm{IC}}$ is due to the sharp change in $d Z_{\mathrm{Fe}} / d t$ between the regimes with and without infall. The fraction of stars in the sharp peak is $10 \%$ for the case of instant infall cessation $(\tau=0$, solid curve). This fraction is even smaller for finite $\tau$ ( $\tau=0.1 \mathrm{Gyr}$, dashed curve; $\tau=0.2 \mathrm{Gyr}$, dot-dashed curve) as more stars are formed after infall starts to decrease. (b) Same as for $(a)$ but for $\tau=1 \mathrm{Gyr}$. The sharp peak at $[\mathrm{Fe} / \mathrm{H}]=-2.2$ is still present but contains only $2 \%$ of the stars and is subdued as compared to the cases of rapid infall cessation ( $\tau \ll 1$ Gyr). The dashed curve assumes a total Fe production rate of $3 \lambda_{\mathrm{Fe}}$ per $\mathrm{H}$ atom in the gas for $[\mathrm{Fe} / \mathrm{H}]>-1$ due to additional Fe contributions from SNe Ia. The sharp drop of the metallicity distribution at $[\mathrm{Fe} / \mathrm{H}]=-1$ corresponds to the onset of SNe Ia. Note that a new broad peak is formed at $[\mathrm{Fe} / \mathrm{H}]=\log (3 \alpha)=-0.8$. Prior to the onset of SNe Ia, $89 \%$ of the baryonic matter has been consumed in star formation. (c) Same as $(a)$ but for a $1 \sigma$ halo. Astration starts at $z_{0}=3.8$, corresponding to $T_{\mathrm{vir}, 0}=10^{4} \mathrm{~K}$, and infall starts to decrease exponentially on a timescale $\tau$ ( $\tau=0$, solid curve; $\tau=1 \mathrm{Gyr}$, dashed curve) at $z_{\mathrm{IC}}=1.6$, corresponding to $M_{\mathrm{IC}}=10^{11} M_{\odot}$. Note that the sharp peak corresponding to the time of infall cessation is shifted to $[\mathrm{Fe} / \mathrm{H}]=-1.8$, compared with $[\mathrm{Fe} / \mathrm{H}]=-2.2$ for the $2 \sigma$ halo. $(d)$ Same as $(a)$ and $(b)$ but for $z_{\mathrm{IC}}=2.8$, corresponding to $M_{\mathrm{IC}}=10^{12} M_{\odot}, \tau=0$, and $\alpha=1 / 6$. Note that the sharp peak corresponding to the time of infall cessation is shifted to [Fe/H] $=-1.9$, compared with $[\mathrm{Fe} / \mathrm{H}]=-2.2$ in $(a)$ and $(b)$, while the broad peak of the solid curve is shifted to $[\mathrm{Fe} / \mathrm{H}]=-0.8$, compared with $[\mathrm{Fe} / \mathrm{H}]=-1.3$ in $(a)$ and $(b)$. Also note that the new broad peak (dashed curve) due to additional Fe contributions from SNe Ia is shifted to $[\mathrm{Fe} / \mathrm{H}]=-0.3$, compared with $[\mathrm{Fe} / \mathrm{H}]=-0.8$ in $(b)$. Prior to the onset of $\mathrm{SNe} \mathrm{Ia}, 45 \%$ of the baryonic matter has been consumed in star formation.

where we have used the estimate for $\lambda_{\text {in }}$ in equation (11) for 1 and $2 \sigma$ halos to obtain the last result. It follows from equations (21) and (22) that the ratio $R_{-/+} \sim 6\left(\mathrm{Gyr} / t_{\mathrm{IC}}\right)^{2 / 3}$ for 1 and $2 \sigma$ halos is much greater than unity for a wide range of $t_{\mathrm{IC}}$. This leads to a sudden drop in $d N / d[\mathrm{Fe} / \mathrm{H}]$ at $t=t_{\mathrm{IC}}$ and produces a sharp peak in the metallicity distribution. The value of $[\mathrm{Fe} / \mathrm{H}]$ at the sharp peak depends on $t_{\mathrm{IC}}$. For a $2 \sigma$ halo with $t_{\mathrm{IC}}=1.4 \mathrm{Gyr}\left(z_{\mathrm{IC}}=4.3\right)$, this peak is at

$$
[\mathrm{Fe} / \mathrm{H}] \sim \log Z_{\mathrm{Fe}}^{\mathrm{QSS}}\left(t_{\mathrm{IC}}\right) \sim-2.2
$$

(see eq. [12]; Fig. 3a).
To compare the effect of instant infall cessation as assumed above with that of a smooth transition for a $2 \sigma$ halo, we have calculated models in which $d(\mathrm{H})_{\text {in }} / d t$ decays smoothly starting at time $t_{\mathrm{IC}}$ with the functional form $\exp \left[-\left(t-t_{\mathrm{IC}}\right) / \tau\right]$ for $t>t_{\mathrm{IC}}$. The results are shown in Figure $3 a$ for $\tau=0.1$ and 0.2 Gyr. As can be seen, the main effect of changing $\tau$ from 0 (instant infall cessation) to a few tenths of a gigayear is to increase the fraction of stars formed at $t>t_{\mathrm{IC}}$. This is because there is more gas available for star formation in the case in which the infall rate decays smoothly over an extended time. However, the width of the sharp peak is increased only slightly from $\sim 0.06$ dex for $\tau=0$ to $\sim 0.14$ dex for $\tau=0.2 \mathrm{Gyr}$. 
Concerning the total population of stars in the metallicity distribution, we note that only $10 \%$ of all the stars are in the sharp peak for $\tau=0$, and this fraction is even smaller for a finite $\tau$. For a slowly decaying infall rate with $\tau \sim 1 \mathrm{Gyr}$, the sharp peak at $t=t_{\mathrm{IC}}$ is still present but contains only $\sim 2 \%$ of the stars and becomes a small feature of an otherwise smooth metallicity distribution (see Fig. $3 b$ for the case of $\tau=1 \mathrm{Gyr}$ ).

The bimodal form of the metallicity distribution of stars discussed above applies to all halos in general. One critical aspect of this bimodal behavior is the value of $[\mathrm{Fe} / \mathrm{H}]$ at the sharp peak. This value is governed by the time $t_{\mathrm{IC}}$ for infall cessation, which depends on the density fluctuation associated with a halo (see $\S 4)$. The metallicity distributions for a $1 \sigma$ halo with instant infall cessation $(\tau=0)$ and smooth transition to exponential decay of infall $(\tau=1 \mathrm{Gyr})$ at a relatively late time of $t_{\mathrm{IC}}=4.1 \mathrm{Gyr}\left(z_{\mathrm{IC}}=1.6\right)$ are shown in Figure $3 c(\alpha=$ $1 / 18$ and $t_{0}=1.6$ Gyr $\left[z_{0}=3.8\right]$ are used). Compared with Figures $3 a$ and $3 b$ for a $2 \sigma$ halo with $t_{\mathrm{IC}}=1.4 \mathrm{Gyr}\left(z_{\mathrm{IC}}=4.3\right)$, the sharp peak for the $1 \sigma$ halo is shifted to a higher $[\mathrm{Fe} / \mathrm{H}]$ of -1.8 . To obtain a peak with a lower $[\mathrm{Fe} / \mathrm{H}]$ of -3 would require $t_{\mathrm{IC}} \sim 0.5 \mathrm{Gyr}$, corresponding to $z_{\mathrm{IC}} \sim 10$ (see eq. [13]). Comparison of Figures $3 a$ and $3 b$ with Figure $3 c$ also shows that the sharp peak for the $2 \sigma$ halo contains a much smaller fraction of stars than that for the $1 \sigma$ halo. Consider the case of instant infall cessation as a specific example. For the $2 \sigma$ halo, the infall rate at high redshift corresponding to $t<t_{\mathrm{IC}}$ is large compared to the astration rate, and only $10 \%$ of stars are formed during the infall phase. In contrast, for the $1 \sigma$ halo, the astration rate is no longer small compared to the infall rate at lower redshift near the end of the infall phase, and $28 \%$ of stars are formed during this phase. From the model presented here, it follows that galaxies in general should have a bimodal distribution of stars, with a relatively small number of stars defining a sharp peak at low $[\mathrm{Fe} / \mathrm{H}]$. This peak reflects the state when gas infall ceased or greatly decreased. Note that we have not addressed the location of stars in a galaxy when discussing their metallicity distribution. The bimodal metallicity distribution is more likely to describe stars in the halo than those in the disk of a galaxy.

As discussed above, the metallicity distribution of stars in a halo is calculated by specifying $\lambda_{\mathrm{Fe}}, \alpha, t_{0}, t_{\mathrm{IC}}$, and the form of infall cessation. The infall rate $\lambda_{\text {in }}$ used in the calculation for $t<t_{\mathrm{IC}}$ is determined from the model of hierarchical structure formation as in $\S 2$. The effects of the form of infall cessation (instant or exponential decay) on the metallicity distribution have been discussed above. To examine the effects of $\alpha$ and $t_{\mathrm{IC}}$, we have calculated the metallicity distribution for a $2 \sigma$ halo with $\alpha=\frac{1}{6}, t_{0}=0.49$ Gyr $\left(z_{0}=9.7\right)$, and instant infall cessation at $t_{\mathrm{IC}}=2.3 \mathrm{Gyr}\left(z_{\mathrm{IC}}=2.8\right)$. This is shown as the solid curve in Figure $3 d$. Compared to the solid curve in Figure $3 a\left(\alpha=1 / 18\right.$ and $\left.t_{\mathrm{IC}}=1.4 \mathrm{Gyr}\left[z_{\mathrm{IC}}=4.3\right]\right)$, the sharp peak in Figure $3 d$ is shifted to $[\mathrm{Fe} / \mathrm{H}]=-1.9 \sim \log Z_{\mathrm{Fe}}^{\mathrm{QSS}}\left(t_{\mathrm{IC}}\right)$ because of a later $t_{\mathrm{IC}}$, and the broad peak (solid curve) in Figure $3 d$ is shifted to $[\mathrm{Fe} / \mathrm{H}]=\log \alpha=-0.8$ because of a larger $\alpha$. In addition, $15 \%$ of all the stars represented by the solid curve in Figure $3 d$ have $-0.5 \lesssim[\mathrm{Fe} / \mathrm{H}] \lesssim 0$, while the corresponding fraction is only $0.3 \%$ for the solid curve in Figure $3 a$. For completeness, we note that $t_{0}$ for the onset of astration has little effect on the metallicity distribution, as most of the stars are formed close to $t=t_{\mathrm{IC}}$ for $t<t_{\mathrm{IC}}$.

It is evident from inspection of Figure 3 that if a protogalactic halo were to expel its gas immediately following the instant cessation of infall, an assemblage of stars with nearly identical $[\mathrm{Fe} / \mathrm{H}]$ would be left behind. This assemblage of stars may be accreted by a larger system and become a globular cluster of the larger system. In this model, the sharp distribution of $[\mathrm{Fe} / \mathrm{H}]$ for the stars in a globular cluster would not be the result of a rapid burst of star formation but rather the result of the following two factors: First, for a protogalactic halo with ongoing infall and astration over an extended period, the rate of star formation would increase rapidly with time and reach a maximum at the time $t_{\mathrm{IC}}$ for instant infall cessation, when there is the largest amount of gas in the halo. This would cause nearly all the stars formed during the infall phase to have the metallicity of the gas at $t=t_{\mathrm{IC}}$. Second, the rapid loss of gas subsequent to infall cessation would terminate further astration. Our model relates the value of $[\mathrm{Fe} / \mathrm{H}]$ for the globular cluster to $t=t_{\mathrm{IC}}$ and therefore can be used to estimate the formation time (relative to the big bang) of the cluster based on its $[\mathrm{Fe} / \mathrm{H}]$ if the initial metallicity of the infalling gas is negligible.

\subsection{General Effects of SNe Ia}

To illustrate the general effects of SNe Ia, we assume that these supernovae start to contribute $\mathrm{Fe}$ when $[\mathrm{Fe} / \mathrm{H}]=-1$ is reached at time $t^{\dagger}$. The rate of Fe production by $\mathrm{SNe}$ Ia is taken to be twice that for SNe II, so that a solar Fe abundance can be achieved over a period of $\sim 10 \mathrm{Gyr}$, as in the Galaxy. The total $\mathrm{Fe}$ production rate per $\mathrm{H}$ atom in the gas then increases from $\lambda_{\mathrm{Fe}}$ at $t<t^{\dagger}$ to $3 \lambda_{\mathrm{Fe}}$ at $t>t^{\dagger}$. Consider halos with instant infall cessation occurring at $t=t_{\mathrm{IC}}$ prior to the onset of SNe Ia (i.e., $t^{\dagger}>t_{\mathrm{IC}}$ ). The evolution of $Z_{\mathrm{Fe}}$ at $t>t^{\dagger}$ for these halos is given by

$$
Z_{\mathrm{Fe}}(t)=Z_{\mathrm{Fe}}\left(t^{\dagger}\right)+3 \lambda_{\mathrm{Fe}}\left(t-t^{\dagger}\right)
$$

where $Z_{\mathrm{Fe}}\left(t^{\dagger}\right)=0.1([\mathrm{Fe} / \mathrm{H}]=-1)$ is taken to correspond to the onset of SNe Ia. The above evolution of $[\mathrm{Fe} / \mathrm{H}]=\log Z_{\mathrm{Fe}}$ is shown for a $1 \sigma$ halo with $t_{0}=1.6 \mathrm{Gyr}\left(z_{0}=3.8\right)$ and $t_{\mathrm{IC}}=4.1 \mathrm{Gyr}\left(z_{\mathrm{IC}}=1.6\right)$ and for a $2 \sigma$ halo with $t_{0}=0.49 \mathrm{Gyr}$ $\left(z_{0}=9.7\right)$ and $t_{\mathrm{IC}}=1.4 \mathrm{Gyr}\left(z_{\mathrm{IC}}=4.3\right)$ in Figure $2 b$. The evolution of $[\mathrm{Fe} / \mathrm{H}]$ at $t>t^{\dagger}$ for the case of no infall is also given by equation (23) and shown in Figure $2 b$. The only effect of SNe Ia on the evolution of $[\mathrm{Fe} / \mathrm{H}]$ for all halos is to increase the rate of Fe enrichment for $t>t^{\dagger}$.

As in the case of infall cessation, the sudden increase of the $\mathrm{Fe}$ production rate per $\mathrm{H}$ atom in the gas at the onset of $\mathrm{SNe} \mathrm{Ia}$ leads to a sharp drop of $d N / d[\mathrm{Fe} / \mathrm{H}]$ at $[\mathrm{Fe} / \mathrm{H}]=-1$. This is shown for a $2 \sigma$ halo with $\alpha=1 / 18$ and a smooth transition to exponential decay of infall $(\tau=1 \mathrm{Gyr})$ at $t_{\mathrm{IC}}=1.4 \mathrm{Gyr}$ $\left(z_{\mathrm{IC}}=4.3\right)$ in Figure $3 b$, and for another $2 \sigma$ halo with $\alpha=$ $1 / 6$ and instant infall cessation at $t_{\mathrm{IC}}=2.3 \mathrm{Gyr}\left(z_{\mathrm{IC}}=2.8\right)$ in Figure $3 d$. For the cases in which only SNe II provide Fe, the metallicity distribution of stars has a broad peak at $[\mathrm{Fe} / \mathrm{H}]=\log \alpha=-1.3$ for Figure $3 b$ and -0.8 for Figure $3 d$ (solid curves). If we include SN Ia contributions for $[\mathrm{Fe} / \mathrm{H}]>$ -1 , this broad peak is truncated, and a new broad peak is formed at $[\mathrm{Fe} / \mathrm{H}]=\log (3 \alpha)=-0.8$ for Figure $3 b$ and -0.3 for Figure $3 d$ (dashed curves). This change to the metallicity distribution is caused by the more rapid increase of $[\mathrm{Fe} / \mathrm{H}]$ with additional contributions from SNe Ia. The above effect of $\mathrm{SNe}$ Ia on the metallicity distribution of stars should apply to all halos. For completeness, we note that the final halo mass for the $2 \sigma$ halo shown in Figure $3 b$ is $5.5 \times 10^{11} M_{\odot}$, corresponding to a baryonic mass of $8.3 \times 10^{10} M_{\odot}$. In this case $89 \%$ of the baryonic matter has been consumed in star formation prior to the onset of SNe Ia. In comparison, the final halo mass for the $2 \sigma$ halo shown in Figure $3 d$ is $10^{12} M_{\odot}$, corresponding to a baryonic mass of $1.5 \times 10^{11} M_{\odot}$. In this case 
$45 \%$ of the baryonic matter has been consumed in star formation prior to the onset of $\mathrm{SNe}$ Ia.

\section{COSMOLOGICAL INPUT FOR HALO EVOLUTION}

In the formal discussion of $\S \S 2$ and 3 on the chemical evolution of a halo, we used the times for the onset of astration $\left(t_{0}\right)$ and infall cessation $\left(t_{\mathrm{IC}}\right)$ and the infall rate $\left(\lambda_{\text {in }}\right)$, all of which are related to the growth of the halo as determined by the CDM model of hierarchical structure formation. We now explicitly explore how this cosmological input is determined. The physical conditions governing the general evolution of a halo are closely related to the mass (mostly in CDM) of the halo. For a halo associated with an $n \sigma$ density fluctuation (an $n \sigma$ halo), its mass $M$ at redshift $z>1$ can be estimated from

$$
n \sigma(M) \approx 1.33(1+z),
$$

where $\sigma(M)$ is the standard deviation of the present power spectrum of overdensity on mass scale $M$. Using the version of equation (24) presented in the Appendix and taking $\sigma(M)$ from Figure 5 of Barkana \& Loeb (2001), we show $M$ as a function of $z$ for 1 and $2 \sigma$ halos, respectively, in Figure $2 a$. The function $M(z)$ for a specific halo was used to calculate the infall rate $\lambda_{\text {in }}$ from equations (6) and (7) in $\S 2$. In addition, when discussing the effects of infall cessation in $\S 3$, we assumed that infall ceases at time $t_{\mathrm{IC}}$, corresponding to redshift $z_{\mathrm{IC}}$, at which the mass of a halo reaches $M\left(z_{\mathrm{IC}}\right)=M_{\mathrm{IC}}$. For $M_{\mathrm{IC}}=10^{11} M_{\odot}, z_{\mathrm{IC}}=1.6$ or 4.3 for a 1 or $2 \sigma$ halo, respectively (see Fig. $2 a$ ). If $M_{\mathrm{IC}}=10^{12} M_{\odot}$ is assumed, then infall cessation is delayed to $z_{\mathrm{IC}}=0.8$ or 2.8 for a 1 or $2 \sigma$ halo, respectively. The redshifts at which infall cessation occurs for other $n \sigma$ halos are given for $M_{\mathrm{IC}}=10^{11}$ and $10^{12} M_{\odot}$, respectively, by the corresponding curves in Figure 4. The range of $M_{\mathrm{IC}}=10^{11}-10^{12} M_{\odot}$ is in accord with the halo masses of typical galaxies.

A critical physical parameter for the onset of astration in a halo is the virial temperature $T_{\text {vir }}$ of its gas. For $z>1$ this temperature is related to the halo mass $M$ as

$$
T_{\mathrm{vir}} \approx 1.04 \times 10^{4}\left(\frac{\mu}{0.6}\right)\left(\frac{M}{10^{8} M_{\odot}}\right)^{2 / 3}\left(\frac{1+z}{10}\right) \mathrm{K}
$$

where $\mu$ is the mean molecular weight and $\mu=1.22$ or 0.6 for a neutral or ionized gas of primordial composition, respectively. Following Barkana \& Loeb (2001), we assume that astration starts in a halo when the gas reaches a threshold virial temperature $T_{\mathrm{vir}, 0}$. At a given redshift $z$, the mass $M=M_{0}$ that is required for a halo to reach $T_{\mathrm{vir}}=T_{\mathrm{vir}, 0}$ can be obtained from equation (25). Using this equation (see the Appendix), we show $M_{0}$ as a function of $z$ for $T_{\mathrm{vir}, 0}=300 \mathrm{~K}$ $(\mu=1.22)$ and $T_{\mathrm{vir}, 0}=10^{4} \mathrm{~K}(\mu=0.6)$, respectively, in Figure $2 a$. The redshift $z=z_{0}$ at which the mass $M(z)$ of an $n \sigma$ halo matches $M_{0}(z)$ then corresponds to the onset of astration $\left(t_{0}\right)$ in this halo. For $T_{\mathrm{vir}, 0}=10^{4} \mathrm{~K}, z_{0}=3.8$ or 9.7 for a 1 or $2 \sigma$ halo, respectively (see Fig. $2 a$ ). If $T_{\mathrm{vir}, 0}=$ $300 \mathrm{~K}$ is assumed, then the onset of astration occurs earlier, at $z_{0}=7.3$ or 16.8 for a 1 or $2 \sigma$ halo, respectively. The redshifts at which astration starts for other $n \sigma$ halos are given for $T_{\mathrm{vir}, 0}=300$ or $10^{4} \mathrm{~K}$, respectively, by the corresponding curves in Figure 4.

The value of $T_{\mathrm{vir}, 0}$ for the onset of astration depends crucially on the available coolant. In the presence of cooling by $\mathrm{H}_{2}$ molecules, $T_{\mathrm{vir}, 0}=300 \mathrm{~K}$ is appropriate. If $\mathrm{H}_{2}$ molecules are dissociated (e.g., Couchman \& Rees 1986; Haiman et al.

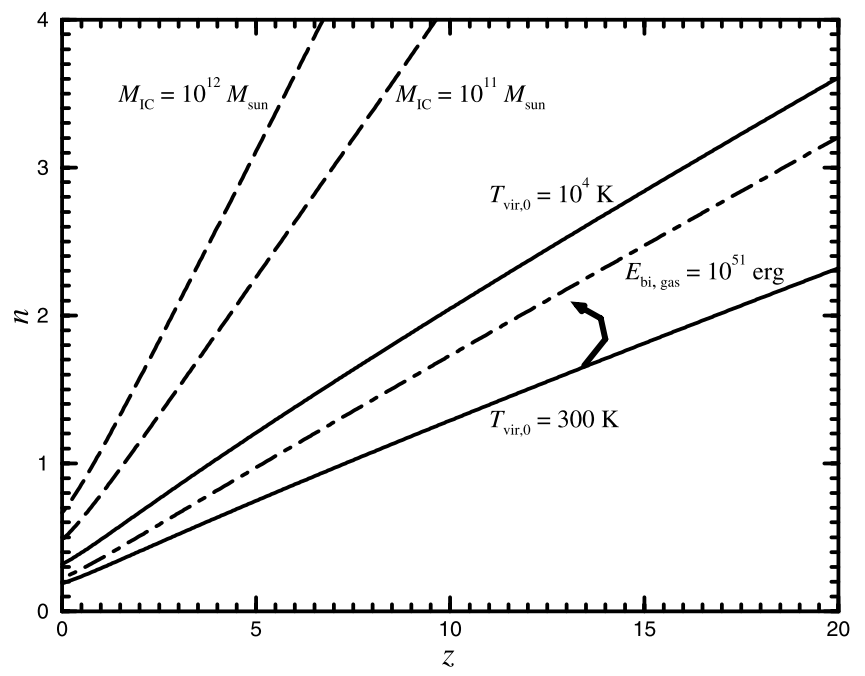

FIG. 4.- Stages of halo evolution. The redshift at which astration starts in an $n \sigma$ halo is given for $T_{\mathrm{vir}, 0}=300$ and $10^{4} \mathrm{~K}$, respectively, by the correspondingly labeled solid curves. The redshift at which an $n \sigma$ halo reaches $E_{\mathrm{bi}, \text { gas }}=10^{51}$ ergs is given by the correspondingly labeled dot-dashed curve. The redshift at which infall ceases for an $n \sigma$ halo is given for $M_{\mathrm{IC}}=10^{11}$ and $10^{12} M_{\odot}$, respectively, by the correspondingly labeled dashed curves. Note that if the onset of astration corresponds to $T_{\mathrm{vir}, 0}=300 \mathrm{~K}$, then a single SN II can expel all the gas from a halo, thereby disrupting its evolution, until $E_{\mathrm{bi} \text {, gas }}=10^{51} \mathrm{ergs}$ is reached. In contrast, if the onset of astration corresponds to $T_{\text {vir }, 0}=10^{4} \mathrm{~K}$, then such a disruption cannot occur, as $E_{\mathrm{bi} \text {, gas }}=10^{51} \mathrm{ergs}$ is always reached prior to the onset of astration. It is expected that $T_{\mathrm{vir}, 0}$ would increase from 300 to $10^{4} \mathrm{~K}$ as $\mathrm{H}_{2}$ molecules are dissociated during the evolution of the universe. Thus, the range of halos that are disrupted by SN II explosions would decrease with time and eventually vanish, as indicated by the curvy arrow.

1997; Ciardi et al. 2000), then $T_{\mathrm{vir}, 0}=10^{4} \mathrm{~K}$ is required for efficient cooling by atomic species. While the choice of $T_{\text {vir }, 0}=300$ or $10^{4} \mathrm{~K}$ does not affect the long-term chemical evolution of a halo (see Fig. 2c), it can make an important difference in the early evolution of the halo. This difference concerns the possibility for a single SN II to expel all the gas, thereby disrupting the chemical evolution of the halo. This possibility can be assessed by comparing the explosion energy of $\sim 10^{51}$ ergs for an SN II with the binding energy $E_{\mathrm{bi}}$,gas of the gas in the halo. For $z>1$ this binding energy is related to the halo mass $M$ as

$$
E_{\mathrm{bi}, \mathrm{gas}} \approx 4.31 \times 10^{52}\left(\frac{M}{10^{8} M_{\odot}}\right)^{5 / 3}\left(\frac{1+z}{10}\right) \text { ergs. }
$$

We consider a simple scenario in which all the gas is expelled when $E_{\mathrm{bi} \text {,gas }}$ for a halo is less than $10^{51}$ ergs. Using equation (26) (see the Appendix), we show the halo mass $M=M_{\mathrm{bi}}$ for $E_{\mathrm{bi}, \text { gas }}=10^{51}$ ergs as a function of $z$ in Figure $2 a$. The redshifts at which different $n \sigma$ halos reach $E_{\mathrm{bi} \text {,gas }}=10^{51} \mathrm{ergs}$ are given by the corresponding curve in Figure 4 . As can be seen from this figure, if $T_{\mathrm{vir}, 0}=300 \mathrm{~K}$ is assumed for the onset of astration, then the chemical evolution of all halos will be disrupted by $\mathrm{SNe}$ II until $E_{\mathrm{bi} \text {,gas }}=10^{51} \mathrm{ergs}$ is reached. In contrast, for $T_{\mathrm{vir}, 0}=10^{4} \mathrm{~K}$, such disruption cannot occur, as $E_{\mathrm{bi} \text {,gas }}=10^{51}$ ergs is always reached prior to the onset of astration.

\section{COMPARISON WITH DATA ON DLA SYSTEMS}

We now compare our chemical evolution model with the data on $[\mathrm{Fe} / \mathrm{H}]$ for DLA systems. Using $T_{\mathrm{vir}, 0}=10^{4} \mathrm{~K}$ for the 


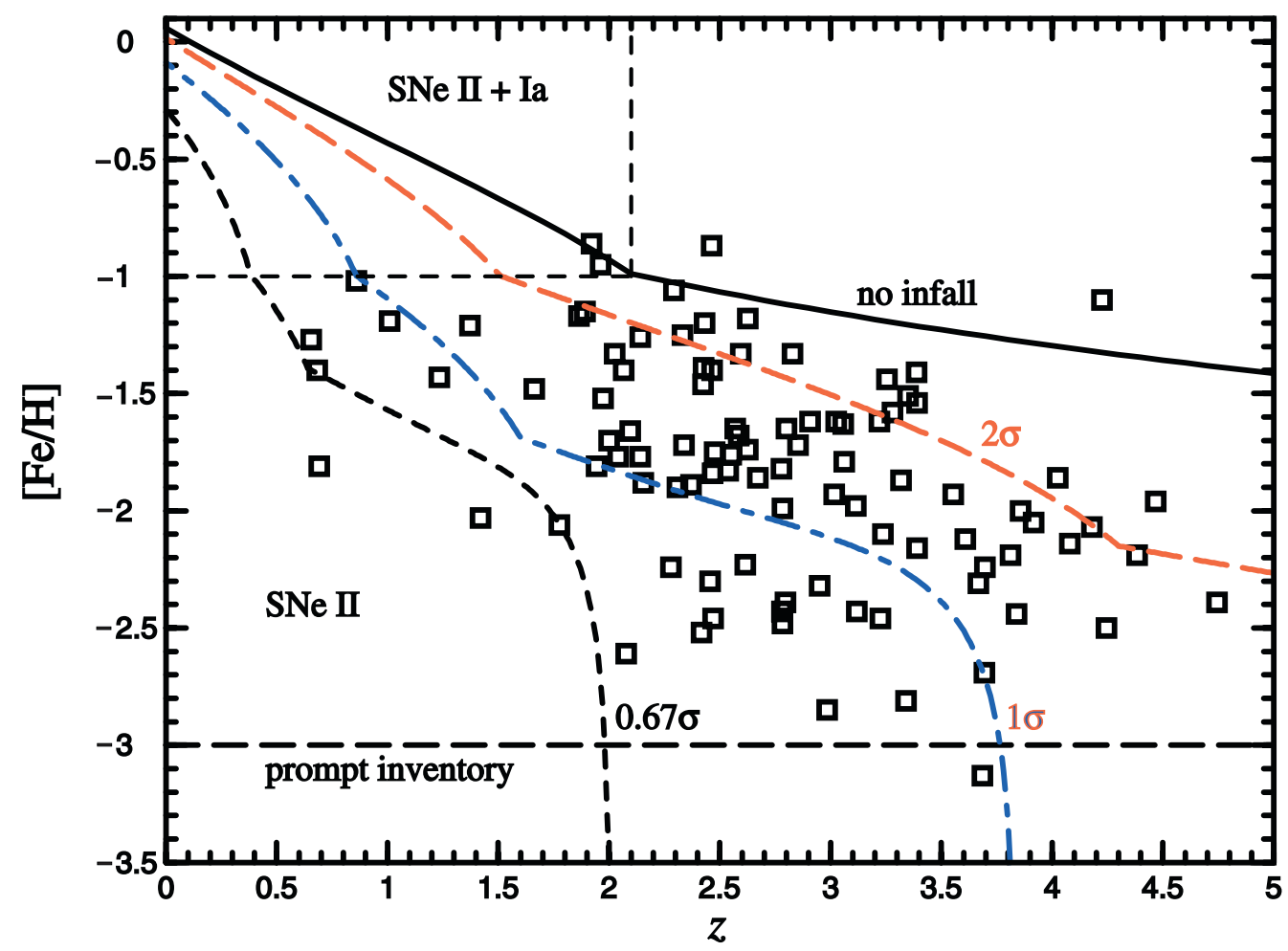

Fig. 5.-Evolution of $[\mathrm{Fe} / \mathrm{H}]$ as a function of $z$ for $0.67 \sigma$ (short-dashed curve), $1 \sigma$ (dot-dashed curve), and $2 \sigma$ (long-dashed curve) halos compared with the data (squares) for 96 DLA systems (Prochaska et al. 2003). The evolution of [Fe/H] for all three halos is calculated using the same assumptions as in Fig. $2 b$, and the dotdashed, long-dashed, and solid curves are the same as in that figure. The prompt inventory proposed earlier (horizontal long-dashed line) is shown for reference.

onset of astration, $M_{\mathrm{IC}}=10^{11} M_{\odot}$ for instant infall cessation, and $[\mathrm{Fe} / \mathrm{H}]=-1$ for the onset of SNe Ia, we show the evolution of $[\mathrm{Fe} / \mathrm{H}]$ as a function of $z$ for $0.67,1$, and $2 \sigma$ halos along with the data on DLA systems (Prochaska et al. 2003) in Figure 5 (see also Qian \& Wasserburg 2003b). The case of no infall is also shown in this figure. It can be seen that the chemical evolution model presented here provides a good description of the data. For any given $z$ the model gives a wide range of $[\mathrm{Fe} / \mathrm{H}]$, corresponding to three different evolutionary stages of DLA systems: sharp initial rise after the onset of astration, QSS due to competition between Fe production and dilution by infall, and uniform growth subsequent to infall cessation. The evolutionary stage of a DLA system at a given $z$ depends on the density fluctuation associated with the system. The large dispersion in $[\mathrm{Fe} / \mathrm{H}]$ for DLA systems at any given $z$ is then accounted for by the different density fluctuations associated with these systems. A detailed quantitative assessment of the model would require many more DLA systems with accurate measurements of $[\mathrm{Fe} / \mathrm{H}]$ at a given $z$ (see Qian \& Wasserburg 2003b for an illustrative example). The model also provides an explanation for the baseline enrichment of $[\mathrm{Fe} / \mathrm{H}] \sim-3$ observed for DLA systems. Subsequent to the onset of astration, it takes only $\sim 30 \mathrm{Myr}$ to reach $[\mathrm{Fe} / \mathrm{H}] \sim-3$ for $\lambda_{\mathrm{Fe}}=(30 \mathrm{Gyr})^{-1}$. Because of this rapid initial rise of $[\mathrm{Fe} / \mathrm{H}]$, the probability of finding DLA systems with $[\mathrm{Fe} / \mathrm{H}]$ below approximately -3 is very low. The baseline enrichment of $[\mathrm{Fe} / \mathrm{H}] \sim-3$ for DLA systems is similar to the abundance level in the IGM that was attributed to a prompt inventory of metals suggested by other arguments (e.g., Wasserburg \& Qian 2000a; Qian \& Wasserburg 2002; Qian et al. 2002). Based on the model presented here, this postulated prompt inventory is not required to explain the data on
DLA systems, although it is also not in conflict with these data.

\section{DISCUSSION AND CONCLUSIONS}

We presented a model for the chemical evolution of the gas and stars in halos as a function of redshift. This model assumes that a normal population of stars including SN II progenitors can form from big bang debris and that SNe II will provide metals such as the elements from the Fe group down to $\mathrm{O}$. If there is a prompt inventory of metals corresponding to $[\mathrm{Fe} / \mathrm{H}] \lesssim-3$ in the IGM, this will simply provide a baseline enrichment for the initial state and will not alter the results. The infall of gas into a halo is assumed to closely follow that of CDM. The infall rate and the general evolution of the halo are prescribed by the model of hierarchical structure formation based on the density fluctuation associated with the halo. For systems that are not disrupted by SN II explosions, the chemical evolution of the gas and stars is explicitly determined by the infall rate as compared with the astration rate and the corresponding rate of metal production by SNe II per $\mathrm{H}$ atom in the gas. Once the astration rate and the metal production rate are specified, the only "free" parameters in this model are the times (or states) at which astration starts and infall ceases for a halo. For all systems not disrupted by SN II explosions, if there is a cessation of gas infall, the metallicities of the stars follow a bimodal distribution. This distribution is characterized by a sharp peak at the value of $[\mathrm{Fe} / \mathrm{H}]$ corresponding to the time of infall cessation and by a broad peak at a higher value of $[\mathrm{Fe} / \mathrm{H}]$ corresponding to the subsequent period of astration, during which the bulk of the remaining gas forms stars. The fraction of stars in the sharp peak depends on how rapidly the infall cessation takes place. 
While it is not clear how observational bias may affect the proper metallicity distribution of stars, we note that the distribution observed for the Galactic halo stars (e.g., Fig. 5 in Christlieb 2003) and the bimodal distribution presented here show a strong resemblance. Based on our model, a distinct narrow peak at low $[\mathrm{Fe} / \mathrm{H}]$ for the Galactic halo stars would signify the time when infall started to decrease for the Galaxy. We also discussed the general effects of $\mathrm{SNe}$ Ia on the chemical evolution of the gas and stars in halos. For $[\mathrm{Fe} / \mathrm{H}] \gtrsim$ -1 the production rate for the Fe group elements is increased because of additional contributions from $\mathrm{SNe}$ Ia. Consequently, the broad peak in the metallicity distribution of stars corresponding to the case in which only SNe II provide Fe is truncated at $[\mathrm{Fe} / \mathrm{H}] \sim-1$, and a new broad peak is formed at a higher $[\mathrm{Fe} / \mathrm{H}]$ value.

We also considered the physical conditions governing the evolution of a halo. The mass $M$ of a halo is an important parameter, and its growth as a function of redshift is determined by the model of hierarchical structure formation based on the $n \sigma$ density fluctuation associated with the halo. We assumed that the cessation of infall for both baryonic and dark matter corresponds to the redshift at which the halo mass reaches $M_{\mathrm{IC}}=10^{11}-10^{12} M_{\odot}$. This range of $M_{\mathrm{IC}}$ is in accord with the halo masses of typical galaxies. The other two important parameters of a halo are the virial temperature $T_{\text {vir }}$ and the binding energy $E_{\mathrm{bi} \text {, gas }}$ of the gas. We assumed that astration starts in a halo when a critical virial temperature $T_{\text {vir, } 0}$ corresponding to a halo mass $M_{0}$ is reached. In the presence of cooling by $\mathrm{H}_{2}$ molecules, $T_{\mathrm{vir}, 0}=300 \mathrm{~K}$ is appropriate, and $M_{0}$ ranges from $\sim 10^{5}$ to $10^{6} M_{\odot}$ for different $n \sigma$ halos. If $\mathrm{H}_{2}$ molecules are dissociated, then $T_{\mathrm{vir}, 0}=10^{4} \mathrm{~K}$ is required, and $M_{0}$ ranges from $\sim 10^{8}$ to $10^{9} M_{\odot}$. While the assumed value of $T_{\text {vir, } 0}$ does not affect the long-term chemical evolution of a halo, it makes an important difference concerning the effect of SN II explosions on the early evolution of the halo. For $T_{\mathrm{vir}, 0}=10^{4} \mathrm{~K}$, astration starts in a halo after the halo mass reaches $M_{\mathrm{bi}}$, corresponding to a gas binding energy of $E_{\mathrm{bi}, \text { gas }}=10^{51} \mathrm{ergs}$. In this case, the chemical evolution of the gas and stars in the halo will not be disrupted by SN II explosions and is summarized in the preceding paragraph. In contrast, for $T_{\mathrm{vir}, 0}=300 \mathrm{~K}$, astration starts in a halo before the halo mass reaches $M_{\mathrm{bi}}$, and all low-mass halos with $M_{0}<$ $M<M_{\mathrm{bi}}$ will have their gas dispersed into the IGM by a single SN II. If such low-mass halos indeed form, then their gas expulsion by SNe II may significantly enrich the IGM at very high redshift. For example, at $z=10$, halos with $M_{0}<$ $M<M_{\text {bi }}$ are associated with 1.3-1.7 $\sigma$ density fluctuations (see Fig. 4), and a fraction

$$
F\left(M_{0}<M<M_{\mathrm{bi}}\right)=\sqrt{2 / \pi} \int_{1.3}^{1.7} \exp \left(-x^{2} / 2\right) d x \approx 10 \%
$$

of all baryonic matter is being processed through such halos. Taking $Z_{\mathrm{Fe}} \sim Z_{\mathrm{Fe}}^{\mathrm{QSS}} \sim 10^{-3}$ (see eq. [12]) for the gas expelled from such halos, we estimate that their gas expulsion would result in an average metallicity of $Z_{\mathrm{Fe}}^{\mathrm{IGM}} \sim F\left(M_{0}<M<\right.$ $\left.M_{\mathrm{bi}}\right) Z_{\mathrm{Fe}}^{\mathrm{QSS}} \sim 10^{-4}$, corresponding to $[\mathrm{Fe} / \mathrm{H}]_{\mathrm{IGM}} \sim-4$ in the IGM at $z=10$. We expect that $T_{\mathrm{vir}, 0}$ for the onset of astration would increase from 300 to $10^{4} \mathrm{~K}$ as various radiation sources turned on during the evolution of the universe. Therefore, the range of low-mass halos that would be disrupted by SN II explosions would decrease with time and eventually vanish. The question of whether such low-mass halos may actually provide significant metal enrichment of the IGM at very high redshift will be discussed in a subsequent paper that considers aspects regarding the dissociation of $\mathrm{H}_{2}$ molecules.

We proposed a possible mechanism of globular cluster formation based on the sharp peak in the metallicity distribution of stars. This peak corresponds to the time of infall cessation. If infall ceases rather suddenly for a protogalactic halo and its gas is rapidly expelled afterward, an assemblage of stars with nearly identical $[\mathrm{Fe} / \mathrm{H}]$ will be left behind. This assemblage of stars may be accreted by a larger system and become a globular cluster of the larger system. To estimate the masses and metallicities of such globular clusters, we assume $T_{\text {vir }, 0}=10^{4} \mathrm{~K}$ for the onset of astration. In this case the gas in a halo will be enriched by SNe II without disruption subsequent to the onset of astration. During the infall phase the amount of infalling gas increases rapidly on a timescale of $\lambda_{\text {in }}^{-1} \sim 0.04[10 /(1+z)]^{5 / 2} \mathrm{Gyr}$ (see eq. [11]). For an astration rate of $d(\mathrm{H})_{\mathrm{as}} / d t=-\left(\lambda_{\mathrm{Fe}} / \alpha\right)(\mathrm{H})$ with $\lambda_{\mathrm{Fe}}=(30 \mathrm{Gyr})^{-1}$ and $\alpha=1 / 18$, the fraction of the halo mass $M$ in stars at redshift $z$ is $f_{*}$ and can be estimated as

$$
f_{*} \sim\left(\Omega_{b} / \Omega_{m}\right)\left(\lambda_{\mathrm{Fe}} / \alpha\right) \lambda_{\text {in }}^{-1} \sim 4 \times 10^{-3}[10 /(1+z)]^{5 / 2} .
$$

If infall ceases at redshift $z$, then the sharp peak in the metallicity distribution contains a total mass $\sim f_{*} M$ of stars with essentially the same $[\mathrm{Fe} / \mathrm{H}]$ as given approximately by $[\mathrm{Fe} / \mathrm{H}]_{\mathrm{QSS}}$ for this $z$ (see eq. [13]). For $T_{\mathrm{vir}, 0}=10^{4} \mathrm{~K}$, the minimum halo mass required for astration $M_{0}=8.1 \times 10^{7}$ and $2.6 \times 10^{8} M_{\odot}$ for $z=10$ and 4, respectively (see Fig. $2 a$ ). Therefore, in the above scenario, the globular clusters would have masses exceeding $f_{*} M_{0} \sim 3 \times 10^{5} M_{\odot}$ and $[\mathrm{Fe} / \mathrm{H}] \sim-3$ if they were formed at $z=10$, while they would have masses exceeding $f_{*} M_{0} \sim 6 \times 10^{6} M_{\odot}$ and $[\mathrm{Fe} / \mathrm{H}] \sim-2.1$ if they were formed at $z=4$. No small globular clusters with masses of $\sim 10^{4} M_{\odot}$ are formed in this scenario unless the larger aggregates are fragmented. We note that the masses derived for Galactic globular clusters are rather uncertain and range from $\sim 300$ to $\sim 10^{7} M_{\odot}$, with a mean of $\sim 2 \times 10^{5} M_{\odot}$ (Mandushev et al. 1991; Richer et al. 1991). The metallicity distribution of Galactic globular clusters is bimodal, with peaks at $[\mathrm{Fe} / \mathrm{H}] \sim-1.6$ and -0.5 , respectively (Armandroff \& Zinn 1988). Approximately $10 \%$ of the clusters have $[\mathrm{Fe} / \mathrm{H}]<-2$. The masses and metallicities of globular clusters in our model are broadly consistent with those of the Galactic population. A detailed comparison requires consideration of possible ranges for $\lambda_{\mathrm{Fe}}, \lambda_{\text {in }}$, and $\alpha$. We also note that stars in an individual Galactic globular cluster typically have a very narrow range of $[\mathrm{Fe} / \mathrm{H}]$, which can be explained by our model. However, there is a considerable range in $[\mathrm{Fe} / \mathrm{H}]$ for the most massive Galactic globular cluster, $\omega$ Centauri (Suntzeff \& Kraft 1996; Norris et al. 1996), with a mass of $7 \times 10^{6} M_{\odot}$ (Richer et al. 1991). The range of [Fe/H] for $\omega$ Cen may have resulted from further astration subsequent to infall cessation based on our model. As an SN II cannot unbind the gas in the protogalactic halo associated with such a large cluster and some additional mechanism for gas loss must be involved, a more extensive evolution of $[\mathrm{Fe} / \mathrm{H}]$ would be expected. The gas loss may have to occur by, e.g., passing through the Galactic disk.

If we assume $T_{\mathrm{vir}, 0}=300 \mathrm{~K}$ for the onset of astration, then a different scenario of globular cluster formation may occur. This scenario is possible if $\mathrm{H}_{2}$ molecules are not dissociated and the IGM is not reionized, so we here only consider it for very high redshift, for instance, $z \sim 10$. For $z=10, M_{0}=1.5 \times 10^{5} M_{\odot}$ 
for the assumed value of $T_{\mathrm{vir}, 0}=300 \mathrm{~K}$ and $M_{\mathrm{bi}}=9.9 \times 10^{6} M_{\odot}$ (see Fig. 2a). A low-mass halo with $M_{0}<M<M_{\text {bi }}$ has started astration, but its gas will be expelled by a single SN II. Since the metals produced by the SN II are expelled along with the gas, any metals in the stars already formed cannot be due to this SN II but must have come from the gas that fell into the halo earlier. In other words, the metallicities of these stars represent the metal inventory of $[\mathrm{Fe} / \mathrm{H}] \lesssim-3$ provided to the IGM at $z \sim 10$ by sources at even higher redshift. For the low-mass halos considered above, the assemblage of stars that remain after the SN II explosion resembles a globular cluster with a mass of $\sim f_{*} M_{0} \sim 500 M_{\odot}$ to $\sim f_{*} M_{\mathrm{bi}} \sim 3 \times 10^{4} M_{\odot}$. We note that Galactic globular clusters in this mass range typically have $[\mathrm{Fe} / \mathrm{H}]$ much higher than -3 . Whether globular clusters in the same mass range but with $[\mathrm{Fe} / \mathrm{H}] \lesssim-3$ exist remains to be seen. The possibility that low-mass halos might form at $z<10$ from an enriched IGM also remains to be explored.

We would like to acknowledge the anonymous referee for one of our earlier studies on cosmochemical evolution (Qian \& Wasserburg 2003a) for being supportive and for suggesting that we might pay attention to infall. Good advice sometimes does fall in. We also want to thank the anonymous referee for the present work and the scientific editor for helpful suggestions. This work was supported in part by DOE grants DE-FG02-87ER40328, DE-FG02-00ER41149 (Y. Z. Q.), and DE-FG03-88ER13851 (G. J. W.), Caltech Division Contribution 8909 (1111).

\section{APPENDIX}

\section{EQUATIONS FOR HALO EVOLUTION}

In this appendix we summarize the equations that are used to calculate the mass $M$, the virial temperature $T_{\text {vir }}$, and the gas binding energy $E_{\mathrm{bi} \text {,gas }}$ of a halo for any redshift $z$. While these equations were discussed in many works, our discussion here closely follows that of Barkana \& Loeb (2001). We consider a flat universe with $\Omega_{m}=0.3, \Omega_{b}=0.045, \Omega_{\Lambda}=0.7$, and $h=0.7$.

The evolution of the mass $M$ for an $n \sigma$ halo is prescribed by

$$
1.686 \frac{D(0)}{D(z)}=n \sigma(M)
$$

where $D(z)$ is the growth factor (Peebles 1980) and $\sigma(M)$ is the standard deviation of the present power spectrum of overdensity on mass scale $M$. The growth factor can be approximated as (e.g., Eisenstein \& Hu 1998)

$$
D(z) \propto \frac{\Omega_{m}^{z}(1+z)^{-1}}{\left(\Omega_{m}^{z}\right)^{4 / 7}-\Omega_{\Lambda}^{z}+\left[1+\left(\Omega_{m}^{z} / 2\right)\right]\left[1+\left(\Omega_{\Lambda}^{z} / 70\right)\right]},
$$

where

$$
\begin{aligned}
\Omega_{m}^{z} & =\frac{\Omega_{m}(1+z)^{3}}{\Omega_{m}(1+z)^{3}+\Omega_{\Lambda}}, \\
\Omega_{\Lambda}^{z} & =\frac{\Omega_{\Lambda}}{\Omega_{m}(1+z)^{3}+\Omega_{\Lambda}} .
\end{aligned}
$$

Note that the proportionality relation in equation (A2) is sufficient to determine the ratio $D(0) / D(z)$ in equation (A1). For $z>1$, equation (A1) can be simplified to give equation (24) for the adopted cosmological parameters.

The virial temperature $T_{\text {vir }}$ of the gas in a halo of mass $M$ at redshift $z$ is

$$
T_{\text {vir }}=1.98 \times 10^{4}\left(\frac{\mu}{0.6}\right)\left(\frac{M}{10^{8} h^{-1} M_{\odot}}\right)^{2 / 3}\left(\frac{\Omega_{m}}{\Omega_{m}^{z}} \frac{\Delta_{c}}{18 \pi^{2}}\right)^{1 / 3}\left(\frac{1+z}{10}\right) \mathrm{K},
$$

where $\Delta_{c}=18 \pi^{2}-82 \Omega_{\Lambda}^{z}-39\left(\Omega_{\Lambda}^{z}\right)^{2}$ (Bryan \& Norman 1998). The binding energy $E_{\mathrm{bi}, \text { gas }}$ of the gas is

$$
E_{\mathrm{bi}, \text { gas }}=5.45 \times 10^{53}\left(\frac{\Omega_{b}}{\Omega_{m}}\right)\left(\frac{M}{10^{8} h^{-1} M_{\odot}}\right)^{5 / 3}\left(\frac{\Omega_{m}}{\Omega_{m}^{z}} \frac{\Delta_{c}}{18 \pi^{2}}\right)^{1 / 3}\left(\frac{1+z}{10}\right) h^{-1} \text { ergs } .
$$

For $z>1$, equations (A5) and (A6) can be simplified to give equations (25) and (26), respectively, for the adopted cosmological parameters.

REFERENCES

Aguirre, A., Schaye, J., Kim, T.-S., Theuns, T., Rauch, M., \& Sargent, W. L. W. 2004, ApJ, 602, 38

Armandroff, T. E., \& Zinn, R. 1988, AJ, 96, 92

Barkana, R., \& Loeb, A. 2001, Phys. Rep., 349, 125

Bromm, V., Ferrara, A., Coppi, P. S., \& Larson, R. B. 2001, MNRAS, 328, 969

Bromm, V., \& Larson, R. B. 2004, ARA\&A, in press

Bryan, G. L., \& Norman, M. L. 1998, ApJ, 495, 80
Burris, D. L., Pilachowski, C. A., Armandroff, T. E., \& Sneden, C. 2000, ApJ, 544,302

Busso, M., Gallino, R., \& Wasserburg, G. J. 1999, ARA\&A, 37, 239

Carney, B. W., Laird, J. B., Latham, D. W., \& Aguilar, L. A. 1996, AJ, 112,668

Christlieb, N. 2003, Rev. Mod. Astron., 16, 191

Christlieb, N., et al. 2002, Nature, 419, 904 
Ciardi, B., Ferrara, A., \& Abel, T. 2000, ApJ, 533, 594

Couchman, H. M. P., \& Rees, M. J. 1986, MNRAS, 221, 53

Eisenstein, D. J., \& Hu, W. 1998, ApJ, 496, 605

Haiman, Z., Rees, M. J., \& Loeb, A. 1997, ApJ, 476, 458

Hartwick, F. D. A. 1976, ApJ, 209, 418

Kobayashi, C., Tsujimoto, T., Nomoto, K., Hachisu, I., \& Kato, M. 1998, ApJ, 503, L155

Lu, L., Sargent, W. L. W., Barlow, T. A., Churchill, C. W., \& Vogt, S. 1996, ApJS, 107, 475

Mandushev, G., Spassova, N., \& Staneva, A. 1991, A\&A, 252, 94

McWilliam, A., Preston, G. W., Sneden, C., \& Searle, L. 1995, AJ, 109, 2757

Norris, J. E., Freeman, K. C., \& Mighell, K. J. 1996, ApJ, 462, 241

Peebles, P. J. E. 1980, The Large-Scale Structure of the Universe (Princeton: Princeton Univ. Press)

Pettini, M., Madau, P., Bolte, M., Prochaska, J. X., Ellison, S. L., \& Fan, X. 2003, ApJ, 594, 695

Prochaska, J. X., Gawiser, E., Wolfe, A. M., Castro, S., \& Djorgovski, S. G. 2003, ApJ, 595, L9

Prochaska, J. X., \& Wolfe, A. M. 2000, ApJ, 533, L5
Prochaska, J. X., \& Wolfe, A. M. 2002, ApJ, 566, 68

Qian, Y.-Z., Sargent, W. L. W., \& Wasserburg, G. J. 2002, ApJ, 569, L61

Qian, Y.-Z., \& Wasserburg, G. J. 2002, ApJ, 567, 515

$$
\text { 2003a, ApJ, 588, } 1099
$$$$
\text { 2003b, ApJ, 596, L9 }
$$

Richer, H. B., Fahlman, G. G., Buonanno, R., Fusi Pecci, F., Searle, L., \& Thompson, I. B. 1991, ApJ, 381, 147

Schaye, J., Aguirre, A., Kim, T.-S., Theuns, T., Rauch, M., \& Sargent, W. L. W. 2003, ApJ, 596, 768

Simcoe, R. A., Sargent, W. L. W., \& Rauch, M. 2002, ApJ, 578, 737 2004, ApJ, 606, 92

Sneden, C., McWilliam, A., Preston, G. W., Cowan, J. J., Burris, D. L., \& Armosky, B. J. 1996, ApJ, 467, 819

Songaila, A. 2001, ApJ, 561, L153

Suntzeff, N. B., \& Kraft, R. P. 1996, AJ, 111, 1913

Tinsley, B. M. 1979, ApJ, 229, 1046

Wasserburg, G. J., \& Qian, Y.-Z. 2000a, ApJ, 529, L21 2000b, ApJ, 538, L99

Westin, J., Sneden, C., Gustafsson, B., \& Cowan, J. J. 2000, ApJ, 530, 783 